\title{
Kodaira dimension and symplectic sums
}

\author{
Michael Usher
}

\begin{abstract}
Modulo trivial exceptions, we show that symplectic sums of symplectic 4-manifolds along surfaces of positive genus are never rational or ruled, and we enumerate each case in which they have Kodaira dimension zero (i.e., are blowups of symplectic 4-manifolds with torsion canonical class). In particular, a symplectic four-manifold of Kodaira dimension zero arises by such a surgery only if it is diffeomorphic to a blowup either of the $K 3$ surface, the Enriques surface, or a member of a particular family of $T^{2}$-bundles over $T^{2}$ each having $b_{1}=2$.
\end{abstract}

Mathematics Subject Classification (2000). Primary 57R17; Secondary 53D35, 57R57.

Keywords. Symplectic Kodaira dimension, symplectic sum, torus fibration, symplectic isotopy.

\section{Introduction}

Our understanding of the diversity of the world of symplectic four-manifolds has been greatly enriched by the introduction in [7] and [18] of the symplectic sum. Given symplectic four-manifolds $\left(X_{1}, \omega_{1}\right),\left(X_{2}, \omega_{2}\right)$ containing embedded, two-dimensional symplectic submanifolds $F_{1} \subset X_{1}, F_{2} \subset X_{2}$ of equal area and genus and an orientation-reversing isomorphism $\Phi: N_{X_{1}} F_{1} \rightarrow N_{X_{2}} F_{2}$ of their normal bundles (which of course exists if and only if $F_{1}$ and $F_{2}$ have opposite self-intersection), the symplectic sum operation provides a natural isotopy class of symplectic structures on the normal connect sum

$$
Z=X_{1} \#_{F_{1}=F_{2}} X_{2}=\left(X_{1} \backslash v_{1}\right) \cup_{\partial \nu_{1} \sim_{\Phi} \partial \nu_{2}}\left(X_{2} \backslash v_{2}\right),
$$

where the $v_{i}$ are tubular neighborhoods of $F_{i}$ and we use the restriction of $\Phi$ to the unit normal circle bundles of the $F_{i}$ to glue the boundaries of the manifolds $X_{i} \backslash v_{i}$. Using the symplectic sum along surfaces of positive genus, various authors over the years have constructed symplectic four-manifolds satisfying an impressive array of properties; see for instance Theorem 6.2 of [7], which for any finitely presented group $G$ gives a number $r(G)$ such that whenever $a+b \equiv 0 \bmod 12$ and $0 \leq a \leq 2(b-$ $r(G))$ there is a symplectic 4-manifold $M_{a, b, G}$ with $\pi_{1}\left(M_{a, b, G}\right)=G, c_{1}^{2}\left(M_{a, b, G}\right)=$ $a$, and $c_{2}\left(M_{a, b, G}\right)=b$. While Gompf's examples were distinguished by their 
classical topological invariants, the symplectic sum also gives rise to infinite families of mutually homeomorphic but nondiffeomorphic symplectic four-manifolds, since if $K$ is a fibered knot the operation of knot surgery with $K$ [3] amounts to a symplectic sum.

The purpose of this note is to show that, notwithstanding the diversity of symplectic four-manifolds that can be constructed via symplectic sum, there are significant topological restrictions on the manifolds that can be obtained in this way. Our results may perhaps best be understood in terms of the notion of (symplectic) Kodaira dimension, a notion which dates from [20] and is discussed in some detail in [14]. We shall recall the definition of Kodaira dimension below. First, recall that a symplectic four-manifold is called minimal if it does not contain any embedded symplectic spheres of square -1 , and that if $(X, \omega)$ is any symplectic four-manifold one may obtain a minimal symplectic four-manifold $\left(X^{\prime}, \omega^{\prime}\right)$ by blowing down a maximal disjoint collection of symplectic $(-1)$-spheres in $X ;\left(X^{\prime}, \omega^{\prime}\right)$ is then called a minimal model for $(X, \omega)$.

Definition 1.1. Let $(X, \omega)$ be a symplectic four-manifold with minimal model $\left(X^{\prime}, \omega^{\prime}\right)$, and let $\kappa_{X^{\prime}} \in H^{2}\left(X^{\prime} ; \mathbb{Z}\right)$ denote the canonical class of $\left(X^{\prime}, \omega^{\prime}\right)$. Then the Kodaira dimension of $(X, \omega)$ is

$$
\kappa(X, \omega)= \begin{cases}-\infty & \text { if } \kappa_{X^{\prime}} \cdot\left[\omega^{\prime}\right]<0 \text { or } \kappa_{X^{\prime}}^{2}<0, \\ 0 & \text { if } \kappa_{X^{\prime}} \cdot\left[\omega^{\prime}\right]=\kappa_{X^{\prime}}^{2}=0, \\ 1 & \text { if } \kappa_{X^{\prime}} \cdot\left[\omega^{\prime}\right]>0 \text { and } \kappa_{X^{\prime}}^{2}=0 \\ 2 & \text { if } \kappa_{X^{\prime}} \cdot\left[\omega^{\prime}\right]>0 \text { and } \kappa_{X^{\prime}}^{2}>0\end{cases}
$$

In Section 2 of [14] and references therein it is shown that $\kappa(X, \omega)$ is well defined for any symplectic four-manifold (in particular it is independent of the choice of minimal model, and one of the four possibilities listed above always holds); coincides with the classical notion of Kodaira dimension in cases when $X$ happens to admit the structure of a complex surface; is equal to $-\infty$ if and only if $X$ is a rational or ruled surface, ${ }^{1}$ and is equal to zero if and only if the canonical class of the minimal model $X^{\prime}$ is torsion. Moreover Theorem 2.6 of [14] shows that the Kodaira dimension $\kappa(M, \omega)$ depends only on the diffeomorphism type of $M$, and not on the symplectic form.

A common feature of the numerous interesting new symplectic four-manifolds that the symplectic sum operation has provided to us is that they have always had Kodaira dimension 1 or 2 . For instance, knot surgery on the K3 surface with a nontrivial fibered knot as in [3] always yields a symplectic four-manifold of Kodaira

\footnotetext{
${ }^{1}$ Here and below we adopt the convention that a ruled surface is a symplectic manifold obtained by (possibly) blowing up the total space of an $S^{2}$-bundle over some Riemann surface. When we want to assume that no blowups have been carried out, we shall refer instead to an " $S^{2}$-bundle."
} 
dimension 1 (even though the result is homeomorphic to the K3 surface, which has Kodaira dimension zero), and (at least aside from some very trivial cases) Gompf's manifolds $M_{a, b, G}$ have Kodaira dimension 1 if $a=0$ and 2 if $a>0$. Our main theorem below will demonstrate that this is not a coincidence. To state it, we make the following definitions.

Definition 1.2. Let $\left(X_{1}, \omega_{1}\right),\left(X_{2}, \omega_{2}\right)$ be symplectic four-manifolds with $F_{i} \subset X_{i}$ embedded symplectic submanifolds of equal area and genus and opposite square.

(1) The symplectic sum $X_{1} \#_{F_{1}=F_{2}} X_{2}$ is called smoothly trivial if, for some $i \in\{1,2\}, X_{i}$ the total space of an $S^{2}$-bundle of which $F_{i}$ is a section. Otherwise, the symplectic sum is called smoothly nontrivial.

(2) The symplectic sum $X_{1} \#_{F_{1}}=F_{2} \quad X_{2}$ is said to be of blowup type if, for some $i \in\{1,2\}$, the pair $\left(X_{i}, F_{i}\right)$ may be obtained from a pair $(E, F)$ consisting of the total space $E$ of an $S^{2}$-bundle of which $F$ is a section by a sequence of zero or more blowups at points not lying on $F$.

Remark 1.3. It is stated without proof in [7] that if $X_{1} \#_{F_{1}=F_{2}} X_{2}$ is a smoothly trivial symplectic sum, say with $\left(X_{2}, F_{2}\right)$ consisting of an $S^{2}$-bundle and a section, then the $\operatorname{sum} X_{1} \#_{F_{1}}=F_{2} X_{2}$ is diffeomorphic to $X_{1}$. It is not difficult to prove this: simply note that $X_{2} \backslash v_{2}$ will be diffeomorphic to a neighborhood of $F_{1}$ in $X_{1}$, so that for at least one choice of the gluing map $\left.\Phi\right|_{\partial \nu_{1}}$ the sum will be diffeomorphic to $X_{1}$; further, the gluing maps $\left.\Phi\right|_{\partial \nu_{1}}: \partial v_{1} \rightarrow \partial v_{2}$ that we are allowed to use in forming the symplectic sum are precisely the restrictions of orientation reversing bundle isomorphisms, and so any two of them differ by precomposing with an orientation preserving diffeomorphism of $\partial v_{1}$ which extends over $v_{1}$, implying therefore that the diffeomorphism type of $X_{1} \#_{F_{1}}=F_{2} X_{2}$ is independent of the gluing map and so is $X_{1}$ in any event. Of course, this argument is dependent on the fact that the gluing map is required to preserve the fibers of the normal circle bundles, an issue which seems to have caused a certain amount of confusion in the literature, where one occasionally finds mistaken claims that symplectic sums with $S^{2}$-bundles along sections sometimes change the diffeomorphism type.

At any rate, the above fact justifies our use of the term "smoothly trivial" to describe such symplectic sums, and by Theorem 2.6 of [14] implies that performing a smoothly trivial symplectic sum leaves the Kodaira dimension unchanged. Incidentally, while these sums are trivial from a smooth standpoint, they generally do alter the symplectic structure in a manner equivalent to the "inflation" technique of [13], a fact which is exploited in [16].

Definition 1.4. If $\left(X_{1}, \omega_{1}\right),\left(X_{2}, \omega_{2}\right)$ are symplectic four-manifolds with $F_{i} \subset X_{i}$ embedded symplectic submanifolds of equal area and genus and opposite square, the symplectic $\operatorname{sum} X_{1} \#_{F_{1}=F_{2}} X_{2}$ is called relatively minimal if for each $i=1,2$, there are no embedded symplectic spheres of square -1 in $X_{i} \backslash F_{i}$. 
Remark 1.5. If a symplectic sum $Z=X_{1} \#_{F_{1}}=F_{2} X_{2}$ is not relatively minimal, then if we blow down maximal disjoint collections of spheres of square -1 in $X_{1} \backslash F_{1}$ and $X_{2} \backslash F_{2}$ to obtain symplectic manifolds $X_{1}^{\prime}, X_{2}^{\prime}$, then the $F_{i}$ survive in the blowdowns and the areas, genera, and self-intersections of the $F_{i}$ are left unchanged. Hence we may form a symplectic sum $Z^{\prime}=X_{1}^{\prime} \#_{F_{1}=F_{2}} X_{2}^{\prime}$, and $Z$ may be recovered from $Z^{\prime}$ by a sequence of blowups. $X_{1}^{\prime} \#_{F_{1}=F_{2}} X_{2}^{\prime}$ will be smoothly trivial if and only if $X_{1} \#_{F_{1}=F_{2}} X_{2}$ is of blowup type. Thus any symplectic 4-manifold which arises as a symplectic sum which is not of blowup type is a blowup of a symplectic 4-manifold which arises as a smoothly nontrivial, relatively minimal symplectic sum. Moreover, symplectic sums which are of blowup type are diffeomorphic to blowups of one of their summands.

Remark 1.6. In this language, the main result of [27] may be rephrased as stating that any symplectic 4-manifold arising as a smoothly nontrivial, relatively minimal symplectic sum along surfaces of positive genus is minimal.

Our main result is the following:

Theorem 1.7. Suppose that $\left(X_{1}, \omega_{1}\right),\left(X_{2}, \omega_{2}\right), F_{1}$, and $F_{2}$ are such that the symplectic sum $Z=X_{1} \#_{F_{1}=F_{2}} X_{2}$ is smoothly nontrivial and relatively minimal and the genus of the $F_{i}$ is positive. Then:

(a) $Z$ does not have Kodaira dimension $-\infty$.

(b) If $Z$ has Kodaira dimension 0 , then the diffeomorphism types of $X_{1}, X_{2}$, and $Z$ are given by one of the rows in the following table, where the notation $M(A, B ; \vec{v})$ denotes a $T^{2}$-bundle over $T^{2}$ as described below or in [24]. Moreover, each entry in the third column of this table can in fact be constructed as a smoothly nontrivial, relatively minimal symplectic sum along a torus.

\begin{tabular}{|c|c|l|}
\hline$X_{1}$ & $X_{2}$ & $\begin{array}{l}\text { possible diffeomorphism types } \\
\text { of } X_{1} \#_{F_{1}}=F_{2} X_{2}\end{array}$ \\
\hline $\mathbb{C} P^{2} \#(18-k) \overline{\mathbb{C} P^{2}}$ & $\mathbb{C} P^{2} \# k \overline{\mathbb{C} P^{2}}$ & $K 3$ surface \\
\hline$S^{2} \times S^{2}$ & $\mathbb{C} P^{2} \# 17 \overline{\mathbb{C} P^{2}}$ & $K 3$ surface \\
\hline $\mathbb{C} P^{2} \#(9-k) \overline{\mathbb{C} P^{2}}$ & $\left(S^{2} \times T^{2}\right) \# k \overline{\mathbb{C} P^{2}}$ & Enriques surface \\
\hline$S^{2} \times S^{2}$ & $\left(S^{2} \times T^{2}\right) \# 8 \overline{\mathbb{C} P^{2}}$ & Enriques surface \\
\hline $\mathbb{C} P^{2} \# 9 \overline{\mathbb{C} P^{2}}$ & $S^{2} \tilde{\times} T^{2}$ & Enriques surface \\
\hline
\end{tabular}




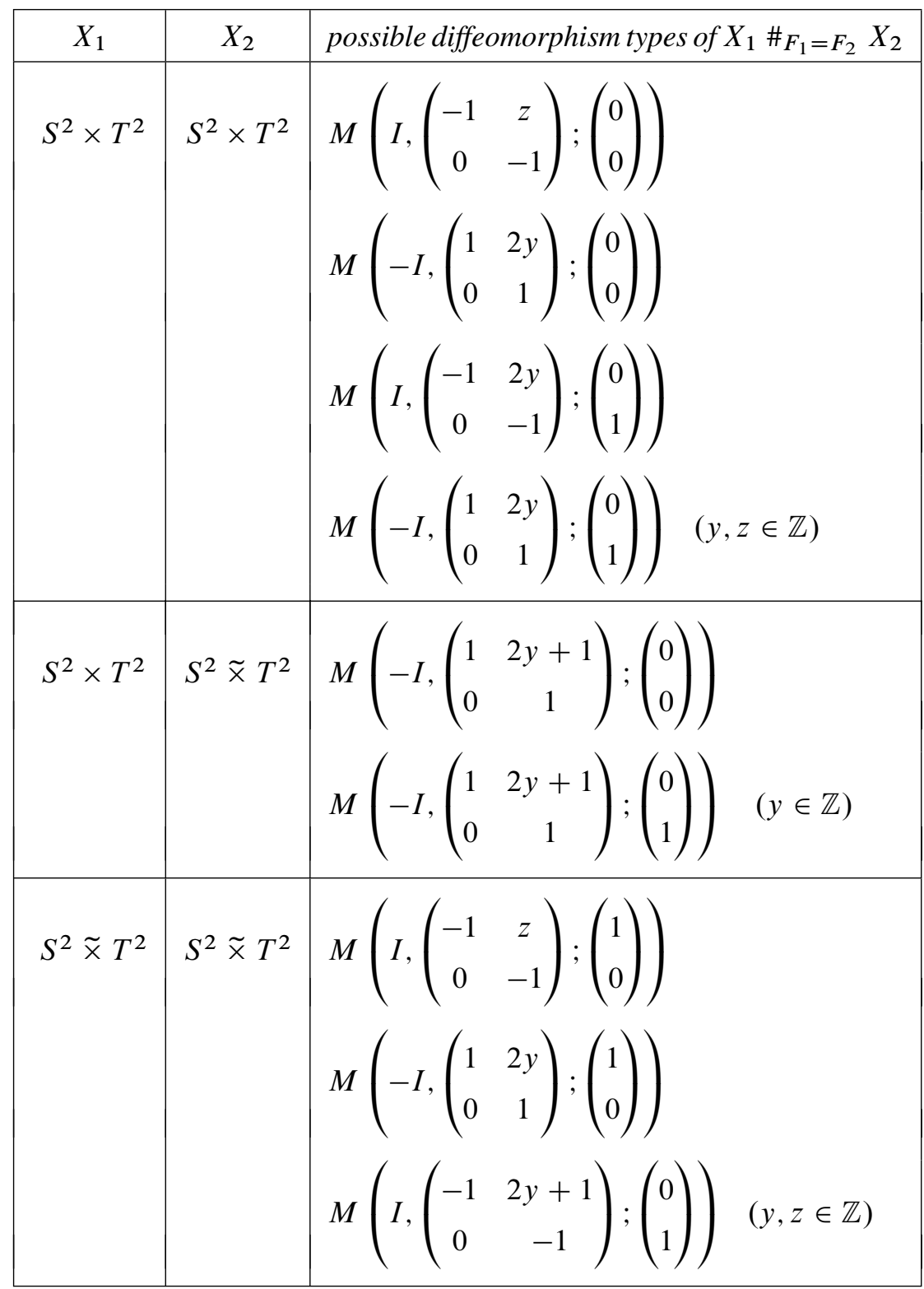

Here $S^{2} \tilde{\times} T^{2}$ refers to the smoothly nontrivial $S^{2}$-bundle over the torus. The $K 3$ surface is, of course, the smooth four-manifold underlying a quartic hypersurface of $\mathbb{C} P^{3}$, while the Enriques surface can be realized either as the quotient of a $K 3$ surface by a free involution which is holomorphic for an appropriate complex structure, or, equivalently, as the result of performing logarithmic transformations of multiplicity 2 
on two fibers of the rational elliptic surface $E(1) ;$ pp. 590-599 of [9] provide a concise description of the complex geometry of these manifolds. The notation $M(A, B ; \vec{v})$ ( $A, B \in \mathrm{SL}(2 ; \mathbb{Z}), \vec{v} \in \mathbb{Z}^{2}, A B=B A$ ) above is as in [24]: identifying $T^{2}=\mathbb{R}^{2} / \mathbb{Z}^{2}$ and writing the coset of $(x, y) \in \mathbb{R}^{2}$ as $\left[\begin{array}{l}x \\ y\end{array}\right]$, we define

$$
\begin{aligned}
M\left(A, B ;\left(\begin{array}{l}
0 \\
0
\end{array}\right)\right)= & \frac{\mathbb{R}^{2} \times T^{2}}{\left(s+1, t,\left[\begin{array}{l}
x \\
y
\end{array}\right]\right) \sim\left(s, t,\left[A\left(\begin{array}{l}
x \\
y
\end{array}\right)\right]\right),}, \\
& \left(s, t+1,\left[\begin{array}{l}
x \\
y
\end{array}\right]\right) \sim\left(s, t,\left[B\left(\begin{array}{l}
x \\
y
\end{array}\right)\right]\right)
\end{aligned}
$$

and let $M(A, B ;(m, n))$ be obtained from $M(A, B ;(0,0))$ by removing a neighborhood of a torus fiber of the projection $[s, t, x, y] \mapsto[s, t]$ and regluing it by the map

$$
\begin{aligned}
\partial D^{2} \times T^{2} & \rightarrow \partial D^{2} \times T^{2}, \\
\left(e^{2 \pi i \theta},[x, y]\right) & \mapsto\left(e^{2 \pi i \theta},[x+m \theta],[y+n \theta]\right) .
\end{aligned}
$$

Since manifolds obtained by general symplectic sums are blowups of those obtained by relatively minimal ones, and since Kodaira dimension is preserved under blowups, Theorem 1.7 has the following corollary:

Corollary 1.8. No symplectic 4-manifold of Kodaira dimension $-\infty$ arises as a symplectic sum along surfaces of positive genus which is not of blowup type, and the only symplectic 4-manifolds of Kodaira dimension 0 which arise as symplectic sums which are not of blowup type along surfaces of positive genus are blowups of symplectic manifolds diffeomorphic to either the K3 surface, the Enriques surface, or a $T^{2}$-bundle over $T^{2}$ having the form $M\left(I,\left(\begin{array}{cc}-1 & z \\ 0 & -1\end{array}\right) ; \vec{v}\right)$ or $M\left(-I,\left(\begin{array}{ll}1 & z \\ 0 & 1\end{array}\right) ; \vec{v}\right)$, where $z \in \mathbb{Z}$ and $\vec{v} \in\{(0,0),(0,1),(1,0)\}$.

Symplectic 4-manifolds of Kodaira dimension $-\infty$ are of course well understood since they are all rational or ruled. In light of Gompf's manifolds $M_{a, b, G}$ mentioned above, the classes of symplectic 4-manifolds of Kodaira dimension either 1 or 2 are each at least as complicated as the class of all finitely presented groups, which have been known to be unclassifiable since the 1950s. The classification of symplectic 4-manifolds of Kodaira dimension zero, meanwhile, is a very interesting open problem. The only presently-known such manifolds are blowups of the $K 3$ surface, the Enriques surface, or an orientable $T^{2}$-bundle over $T^{2}$ (all of the latter were proven to admit symplectic structures in [5]), and a recent striking result independently proven in [1] and [15] implies that any minimal symplectic 4-manifold of Kodaira dimension zero necessarily has the same rational homology as one of the known minimal examples. Theorem 1.7 thus shows that (up to diffeomorphism) if any new symplectic 
4-manifolds of Kodaira dimension zero do exist, then they cannot be found by performing symplectic sums along surfaces of positive genus. Note that since blowing down a (-1)-sphere amounts to performing a symplectic sum with $\mathbb{C} P^{2}$ along a line, any symplectic 4-manifold arises (in a somewhat trivial fashion) as a smoothly nontrivial symplectic sum along spheres. It would be interesting to know whether or not new symplectic 4-manifolds of Kodaira dimension zero can arise by symplectic sum with $\mathbb{C} P^{2}$ along a quadric (i.e., by blowing down a (-4)-sphere), or more generally whether or not new symplectic 4-manifolds of Kodaira dimension zero can arise via the rational blowdown procedure of [2].

Theorem 1.7 pins down the diffeomorphism type of the symplectic sums in question; it would be somewhat preferable to have a result specifying the symplectic deformation type (though it is worth noting that this may be a moot point, since any two symplectic structures on any of the manifolds in the last column of the table in Theorem 1.7 have the same canonical class, and there are not currently any known examples of smooth four-manifolds admitting deformation inequivalent symplectic structures that have the same canonical class). In the case that the symplectic sum is a $T^{2}$-bundle over $T^{2}$, our proof can be seen to imply that the symplectic form will be positive on the fibers, and so will be deformation equivalent to a form obtained by the Thurston trick. When the sum is diffeomorphic to the $K 3$ surface or the Enriques surface, the situation is perhaps more subtle, since in our proofs we use, among other things, the fact that any orientation-preserving diffeomorphism of the boundary of the complement of a tubular neighborhood of a fiber in the rational elliptic surface $E(1)$ extends to the whole complement, and so since such a diffeomorphism cannot always be made symplectic we lose control of the symplectic form.

We should mention that, as in Remark 1.3, although the conclusion of Theorem 1.7 only concerns diffeomorphism types the result is sensitive to the fact that the sum operation is symplectic and so the gluing map $\Phi: \partial v_{1} \rightarrow \partial v_{2}$ is required to be the restriction of an anti-isomorphism of complex line bundles (rather than just an arbitrary diffeomorphism). Of course, if $\Phi$ were replaced by an arbitrary diffeomorphism, the sum might not admit a symplectic structure; for instance for $n \geq 2$ a degree-zero logarithmic transformation of a fiber of the elliptic surface $E(n)$ (which results from an appropriate gluing of the complement of the symplectic torus fiber of $E(n)$ to the complement of a symplectic torus in $\left.T^{2} \times S^{2}\right)$ is diffeomorphic to the non-symplectic manifold $(2 n-1) \mathbb{C} P^{2} \#(10 n-1) \overline{\mathbb{C} P^{2}}$ (Theorem 6.1 of [6]). In other cases, though, a more general gluing map gives rise to a manifold that happens to admit a symplectic structure, which may even have Kodaira dimension zero: for instance, any of the $T^{2}$-bundles over $T^{2}$ given in our notation above as $M(I, I ;(m, n))$ may be obtained by an appropriate gluing of the complement of a symplectic torus in $T^{4}$ to the complement of a symplectic torus in $T^{2} \times S^{2}$; these all admit symplectic structures by [5] (or indeed by earlier work; the case $(m, n)=(0,1)$ is the Kodaira-Thurston manifold) with trivial canonical class and so Kodaira dimension zero. However, for 
$(m, n) \neq(0,0), b_{1}(M(I, I ;(m, n)))=3$, whereas all of the $T^{2}$-bundles over $T^{2}$ appearing in Theorem 1.7 have $b_{1}=2$, so none of the manifolds $M(I, I ;(m, n))$ occur as smoothly nontrivial symplectic sums.

In the next section, we prove part (a) of Theorem 1.7, which might best be seen as a corollary of the arguments of [27]. The rest of the paper consists of the proof of part (b), which relies on a result describing the behavior of the canonical class of a symplectic sum in order to reduce the result to a small number of cases which can be dispensed with in turn. A useful tool in two of the cases is Lemma 3.3, which states that if $F$ is a symplectic torus in $\mathbb{C} P^{2} \# 9 \overline{\mathbb{C} P^{2}}$ Poincaré dual to the first Chern class, then there is a diffeomorphism of pairs taking $\left(\mathbb{C} P^{2} \# 9 \overline{\mathbb{C} P^{2}}, F\right)$ to the pair consisting of a rational elliptic surface together with one of its regular fibers. This should be contrasted with various non-isotopy results for symplectic tori Poincaré dual to multiples of the first Chern class, e.g., in [4].

Acknowledgement. I am grateful for the referee's corrections and suggestions. This work was partially supported by an NSF Postdoctoral Fellowship.

\section{Rational and ruled surfaces}

Part (a) of Theorem 1.7 is an easy consequence of two results proven in [27]. On the one hand, from Section 2 of that paper, we find (based largely on [12]), that if $Z=X_{1} \#_{F_{1}=F_{2}} X_{2}$ is a symplectic sum, and if $A \in H_{2}(Z ; \mathbb{Z})$ is any nonzero homology class having a nontrivial Gromov-Witten invariant counting genus-zero holomorphic curves, then there are classes $A_{1} \in H_{2}\left(X_{1} ; \mathbb{Z}\right), A_{2} \in H_{2}\left(X_{2} ; \mathbb{Z}\right)$, each represented by a union of genus zero stable maps which are pseudoholomorphic for some almost complex structure $J_{i}$ on $X_{i}(i=1,2)$ which preserves $T F_{i}$ such that

$$
\left\langle\kappa_{X_{1}}+\mathrm{PD}\left[F_{1}\right], A_{1}\right\rangle+\left\langle\kappa_{X_{2}}+\mathrm{PD}\left[F_{2}\right], A_{2}\right\rangle=\left\langle\kappa_{Z}, A\right\rangle<0,
$$

where the final inequality results from the fact that the expected dimension of genuszero pseudoholomorphic representatives of $A$, namely $-1-\left\langle\kappa_{Z}, A\right\rangle$, must be nonnegative in order for the corresponding Gromov-Witten invariant to be nonvanishing.

On the other hand, Proposition 3.9 of [27], translated into the terminology of the introduction, states that if the symplectic sum $Z=X_{1} \#_{F_{1}=F_{2}} X_{2}$ is smoothly nontrivial and relatively minimal, and if the $F_{i}$ have positive genus, then the $F_{i}$ are "rationally $K$-nef," which is to say, for $i=1,2$, if $A \in H_{2}\left(X_{i} ; \mathbb{Z}\right)$ is represented by a $J_{i}$-holomorphic sphere for some almost complex structure $J_{i}$ preserving $T F_{i}$, then we necessarily have $\left\langle\kappa_{X_{i}}+\mathrm{PD}\left[F_{i}\right], A\right\rangle \geq 0$.

As such, if the symplectic sum is smoothly nontrivial and relatively minimal, and if the genus of the surfaces involved is positive, then (1) cannot hold, and so $Z$ cannot admit any nonvanishing genus zero Gromov-Witten invariants in any nontrivial homology classes. In [27] this was used to conclude that $Z$ is minimal; in 
our present case, we simply note that any rational or ruled surface does admit a nonvanishing genus-zero Gromov-Witten invariant in a nontrivial homology class (the proper transform of the hyperplane class for blowups of $\mathbb{C} P^{2}$, or the class of the fiber of a ruling for ruled surfaces; see, e.g., [19]). Thus rational and ruled surfaces (i.e., symplectic 4-manifolds of Kodaira dimension $-\infty$ ) cannot arise as smoothly nontrivial, relatively minimal symplectic sums along surfaces of positive genus, proving part (a) of Theorem 1.7.

\section{4-manifolds of Kodaira dimension zero}

The proof of part (b) of Theorem 1.7 depends on the following result.

Theorem 3.1. Let $\left(X_{1}, \omega_{1}\right),\left(X_{2}, \omega_{2}\right)$ be symplectic 4-manifolds, $F_{i} \subset X_{i}(i=1,2)$ embedded symplectic submanifolds of equal positive genus, equal area, and opposite self-intersection, and $\left(Z=X_{1} \#_{F_{1}}=F_{2} X_{2}, \omega\right)$ the symplectic sum of the $X_{i}$ along the $F_{i}$. Assume that the symplectic sum is smoothly nontrivial and that $(Z, \omega)$ has Kodaira dimension zero and is minimal. Then the $F_{i}$ are both tori, and are Poincaré dual to $-\kappa_{X_{i}}$.

Proof. According to Theorem 2.1 of [12], there is a symplectic 6-manifold $(\mathcal{Z}, \Omega)$ equipped with a projection $\pi: \mathcal{Z} \rightarrow D^{2}$ with the property that, for $\lambda \neq 0$, $\left(\pi^{-1}(\lambda),\left.\Omega\right|_{\pi^{-1}(\lambda)}\right)$ is isotopic to the symplectic sum $Z$, while $\left(\pi^{-1}(0),\left.\Omega\right|_{\pi^{-1}(0)}\right)$ is the singular symplectic manifold obtained by directly gluing the $X_{i}$ along the $F_{i}$, so that the two pieces intersect in a copy $F$ of the $F_{i}$. As seen in the proof of Lemma 2.2 of [12], one has (for $\lambda \neq 0$ ) $\left.\kappa_{\mathcal{Z}}\right|_{\pi^{-1}(\lambda)}=\kappa_{\pi^{-1}(\lambda)}$, while for $i=1,2$ $c_{1}\left(\left.T Z\right|_{X_{i}}\right)=c_{1}\left(X_{i}\right)-\mathrm{PD}_{X_{i}}\left[F_{i}\right]$.

We claim now that, where $i_{\lambda}: \pi^{-1}(\lambda) \rightarrow \mathcal{Z}, i_{j}: X_{j} \rightarrow \mathbb{Z}(j=1,2)$ are the inclusions, $i_{\lambda *}\left[\pi^{-1}(\lambda)\right]$ is equal to $i_{1 *}\left[X_{1}\right]+i_{2 *}\left[X_{2}\right]$ in $H_{4}(\mathbb{Z} ; \mathbb{Z})$. This essentially follows from the description of $Z$ in Section 2 of [12]. Namely, as seen there, $\pi^{-1}(\lambda)$ for $0<\lambda \ll 1$ may (up to isotopy) be obtained from $\pi^{-1}(0)=X_{1} \cup_{F} X_{2}$ as follows: a neighborhood of $F \subset \mathcal{Z}$ is diffeomorphic to a neighborhood $U$ of the zero section in $L \oplus L^{*} \rightarrow F$ where $L \rightarrow F$ is a complex line bundle of degree $\left[F_{1}\right]^{2}$ and $L^{*}$ is its dual; putting a Hermitian metric $|\cdot|$ on $L$ (which then induces one on $\left.L^{*}\right)$ and arranging that $U=\left\{(\alpha, v, x) \in L \oplus L^{*}|| \alpha|| v \mid, \leq 1\right\}$, up to smooth isotopy $\pi^{-1}(\lambda)$ coincides with $\pi^{-1}(0)$ outside $U$ and its intersection with $U$ is given by $\left\{(\alpha, v, x) \in L \oplus L^{*} \mid\langle\alpha, v\rangle=\chi(|\alpha|+|v|) \lambda\right\}$ where $\chi:[0,1] \rightarrow[0,1]$ is a bump function supported in $\left[0,2 \lambda^{1 / 4}\right]$ and equal to 1 on $\left[0, \lambda^{1 / 4}\right]$. Observe that $X_{1}$ is homologous to the chain obtained by replacing $X_{1} \cap U$ by (in hopefully self-explanatory notation)

$$
\left\{(\bar{v}, v, x)|| v \mid \leq \lambda^{1 / 2}\right\}+\left\{(\alpha, v, x) \in \pi^{-1}(\lambda)\left|\lambda^{1 / 2} \leq\right| v \mid \leq 1\right\}
$$


and likewise $X_{2}$ is homologous to the chain obtained by replacing $X_{2} \cap U$ by

$$
\left\{(\alpha, \bar{\alpha}, x)|| \alpha \mid \leq \lambda^{1 / 2}\right\}+\left\{(\alpha, v, x) \in \pi^{-1}(\lambda)\left|\lambda^{1 / 2} \leq\right| \alpha \mid \leq 1\right\} .
$$

But adding these two chains together and passing to homology just recovers $i_{\lambda *}\left[Z_{\lambda}\right]$ (as the first terms in the two expressions above now cancel), verifying that indeed $i_{\lambda *}\left[\pi^{-1}(\lambda)\right]=i_{1 *}\left[X_{1}\right]+i_{2 *}\left[X_{2}\right] \in H_{4}(\mathbb{Z} ; \mathbb{Z})$.

As such, we have

$$
\begin{aligned}
\left\langle\kappa_{Z} \cup[\omega],[Z]\right\rangle= & \left\langle i_{\lambda}^{*}\left(\kappa_{\mathcal{Z}} \cup[\Omega]\right),\left[\pi^{-1}(\lambda)\right]\right\rangle=\left\langle\kappa_{\mathcal{Z}} \cup[\Omega], i_{\lambda *}\left[\pi^{-1}(\lambda)\right]\right\rangle \\
= & \left\langle\kappa_{\mathcal{Z}} \cup[\Omega], i_{1 *}\left[X_{1}\right]\right\rangle+\left\langle\kappa_{\mathcal{Z}} \cup[\Omega], i_{2 *}\left[X_{2}\right]\right\rangle \\
= & \left\langle\kappa_{\mathcal{Z}}\left|X_{1} \cup[\Omega]\right|_{X_{1}},\left[X_{1}\right]\right\rangle+\left\langle\kappa_{\mathcal{Z}}\left|X_{2} \cup[\Omega]\right|_{X_{2}},\left[X_{2}\right]\right\rangle \\
= & \left\langle\left(\kappa_{X_{1}}+\operatorname{PD}_{X_{1}}\left[F_{1}\right]\right) \cup\left[\omega_{1}\right],\left[X_{1}\right]\right\rangle \\
& \quad+\left\langle\left(\kappa_{X_{2}}+\operatorname{PD}_{X_{2}}\left[F_{2}\right]\right) \cup\left[\omega_{2}\right],\left[X_{2}\right]\right\rangle \\
= & \left\langle\kappa_{X_{1}} \cup\left[\omega_{1}\right],\left[X_{1}\right]\right\rangle+\left\langle\kappa_{X_{2}} \cup\left[\omega_{2}\right],\left[X_{2}\right]\right\rangle+\int_{F_{1}} \omega_{1}+\int_{F_{2}} \omega_{2} .
\end{aligned}
$$

Note that nothing that we have done so far makes any use of the assumption that $(Z, \omega)$ is minimal with Kodaira dimension zero. However, when we do implement that assumption, the left hand side of (2) becomes zero. Hence

$$
\left(\left\langle\kappa_{X_{1}} \cup\left[\omega_{1}\right],\left[X_{1}\right]\right\rangle+\int_{F_{1}} \omega_{1}\right)+\left(\left\langle\kappa_{X_{2}} \cup\left[\omega_{2}\right],\left[X_{2}\right]\right\rangle+\int_{F_{2}} \omega_{2}\right)=0 .
$$

We claim now that each $\kappa_{X_{i}}$ is proportional to $\operatorname{PD}\left[F_{i}\right]$ in $H^{2}\left(X_{i} ; \mathbb{R}\right)$. Indeed, suppose that this were not the case, say for $i=1$. Then we could find an element $\beta \in$ $H^{2}\left(X_{1} ; \mathbb{R}\right)$ such that $\left\langle\kappa_{X_{1}} \cup \beta,\left[X_{1}\right]\right\rangle=1$ but $\left\langle\beta,\left[F_{1}\right]\right\rangle=0$. Moreover, since small closed perturbations of symplectic forms are still symplectic, for any sufficiently small $\varepsilon>0$ the cohomology class $\left[\omega_{1}\right]+\varepsilon \beta$ would admit a symplectic form, say $\omega_{1}^{\varepsilon}$, which (assuming $\varepsilon$ is small enough) would continue to make $F_{1}$ symplectic, and would induce the same canonical class $\kappa_{X_{1}}$ as does $\omega_{1}$ by virtue of being deformation equivalent to $\omega_{1}$. We would then have

$$
\int_{F_{1}} \omega_{1}^{\varepsilon}=\int_{F_{1}} \omega_{1}, \quad\left\langle\kappa_{X_{1}} \cup\left[\omega_{1}^{\varepsilon}\right],\left[X_{1}\right]\right\rangle=\left\langle\kappa_{X_{1}} \cup\left[\omega_{1}\right],\left[X_{1}\right]\right\rangle+\varepsilon .
$$

But then we could apply the symplectic sum operation to the symplectic manifolds $\left(X_{1}, \omega_{1}^{\varepsilon}\right),\left(X_{2}, \omega_{2}\right)$ along the $F_{i}$ to obtain a new symplectic manifold $\left(Z^{\varepsilon}, \omega^{\varepsilon}\right)$, diffeomorphic (indeed deformation equivalent) to $(Z, \omega)$, and by (2) we would have

$$
\left\langle\kappa_{Z^{\varepsilon}} \cup[\omega],\left[Z^{\varepsilon}\right]\right\rangle=\varepsilon>0 .
$$


So $\left(Z^{\varepsilon}, \omega^{\varepsilon}\right)$ is not a minimal symplectic manifold of Kodaira dimension zero. But by Theorem 2.6 of [14], the Kodaira dimension of a symplectic four-manifold is a diffeomorphism invariant, so since $Z^{\varepsilon}$ is diffeomorphic to $Z$ (and since the minimality or nonminimality of $Z$ is also a diffeomorphism invariant; see, e.g., Proposition 2.1 of [14]) this is impossible. This contradiction shows that, for some $\mu_{1}, \mu_{2} \in \mathbb{R}$, we have $\operatorname{PD}\left(\kappa_{X_{i}}\right)=\mu_{i}\left[F_{i}\right] \in H_{2}(X ; \mathbb{R})$ for $i=1,2$.

Evidently, since $\left\langle\kappa_{Z} \cup[\omega],[Z]\right\rangle=0$, we must have

$$
\begin{aligned}
\left(2+\mu_{1}+\mu_{2}\right) \int_{F_{1}} \omega_{1}= & \left(\left\langle\kappa_{X_{1}} \cup\left[\omega_{1}\right],\left[X_{1}\right]\right\rangle+\int_{F_{1}} \omega_{1}\right) \\
& +\left(\left\langle\kappa_{X_{2}} \cup\left[\omega_{2}\right],\left[X_{2}\right]\right\rangle+\int_{F_{2}} \omega_{2}\right)=0,
\end{aligned}
$$

so since $\int_{F_{1}} \omega_{1}>0$ we have

$$
\mu_{1}+\mu_{2}=-2 .
$$

In particular, at least one of the $\mu_{i}$, say $\mu_{1}$, is negative. Then $\left\langle\kappa_{X_{1}} \cup\left[\omega_{1}\right],\left[X_{1}\right]\right\rangle=$ $\mu_{1} \int_{F_{1}} \omega_{1}<0$, so by Theorem B of [17] and results of [26] $\left(X_{1}, \omega_{1}\right)$ must be either a rational surface or an irrational ruled surface.

Now if $X_{1}=\mathbb{C} P^{2} \# k \overline{\mathbb{C} P^{2}}$, then where $H$ is the hyperplane class and $E_{1}, \ldots, E_{k}$ are the classes of the exceptional spheres of the blowups, we have $\operatorname{PD}\left(\kappa_{X_{1}}\right)=-3 H+$ $\sum_{i=1}^{k} E_{i}$. Since $P D\left(\kappa_{X_{1}}\right)$ is primitive and the embedded surface $F_{1}$ represents $\frac{1}{\mu_{1}} P D\left(\kappa_{X_{1}}\right)$, we have $\frac{1}{\mu_{1}} \in \mathbb{Z}$ and so $\mu_{1} \geq-1$. Similarly if $X_{1}=S^{2} \times S^{2}$ then $\mathrm{PD}\left(\kappa_{X_{1}}\right)=-2\left[S^{2} \times\{\mathrm{pt}\}\right]-2\left[\{\mathrm{pt}\} \times S^{2}\right]$, and again our assumptions on $F_{1}$ force $\mu_{1} \geq-1$ ( $\mu_{1}=-2$ is ruled out both by the assumption that $F_{1}$ has positive genus and by the assumption that the sum is smoothly nontrivial, so that $F_{1}$ is not a section of an $S^{2}$-bundle). Thus if $X_{1}$ is rational then $\mu_{1} \geq-1$.

Suppose now that $X_{1}$ is an irrational ruled surface; say $X_{1}=\left(S^{2} \times \Sigma_{h}\right) \# k \overline{\mathbb{C} P^{2}}$ ( $h \geq 1, k \geq 0$ ). Then where $\sigma$ is the homology class of the proper transform of a section of $S^{2} \times \Sigma_{h} \rightarrow \Sigma_{h}$ and $f$ is the class of the proper transform of a generic fiber, we have $\operatorname{PD}\left(\kappa_{X_{1}}\right)=-2 \sigma+(2 h-2) f+\sum_{i=1}^{k} e_{i}$. If $k>0$, then in order for $\left[F_{1}\right]$ to be an integral class we necessarily have $\mu_{1} \geq-1$. If $k=0$, we have either $\mu_{1} \geq-1$ or $\mu_{1}=-2$, but if $\mu_{1}=-2$ then by Proposition 3.3 of [27] there is a ruling on $X_{1}$ of which $F_{1}$ is a section, which is forbidden by the assumption that the sum is smoothly nontrivial. Finally, if $X_{1}$ is a nontrivial $S^{2}$-bundle $S^{2} \tilde{\times} \Sigma_{h}$ over a surface of genus $h>0$, then where $s^{+}, s^{-}$are sections of square +1 and -1 respectively, we have $\mathrm{PD}\left(\kappa_{X}\right)=(2 h-3)\left[s^{+}\right]-(2 h-1)\left[s^{-}\right]$, so that since $\left[F_{1}\right]=\frac{1}{\mu_{1}} \mathrm{PD}\left(\kappa_{X}\right) \in H_{2}\left(X_{1} ; \mathbb{Z}\right)$, we get $\left|\frac{1}{\mu_{1}}\right| \geq 1$, so $\mu_{1} \geq-1$.

Summing up, we have shown that since $\mu_{1}<0, X_{1}$ is rational or ruled, and that (because $X_{1}$ is rational or ruled) by the hypothesis of the theorem we have $\mu_{1} \geq-1$. But then since $\mu_{1}+\mu_{2}=-2$, we must have $\mu_{2}<0$, which then forces $X_{2}$ to be 
rational or ruled, and so by the same arguments $\mu_{2} \geq-1$. So since $\mu_{1}+\mu_{2}=-2$ and $\mu_{1}, \mu_{2} \geq-1$, we in fact have $\mu_{1}=\mu_{2}=-1$, i.e., $\left[F_{i}\right]=-\operatorname{PD}\left(\kappa_{X_{i}}\right)$ for $i=1,2$.

To see that the $F_{i}$ are tori, simply note that the adjunction formula gives

$$
2 g\left(F_{i}\right)-2=\left[F_{i}\right]^{2}+\left\langle\kappa_{X_{i}},\left[F_{i}\right]\right\rangle=\kappa_{X_{i}}^{2}-\kappa_{X_{i}}^{2}=0 .
$$

Theorem 3.1 and its proof show that, in order for a smoothly nontrivial symplectic sum $X_{1} \#_{F_{1}}=F_{2} X_{2}$ along a surface of positive genus to be minimal and of Kodaira dimension zero, the $X_{i}$ necessarily are rational or ruled, and the $F_{i}$ are tori Poincaré dual to $-\kappa_{X_{i}}$. The main theorem of [27] shows that the sum will automatically be minimal provided that the $X_{i}$ and $F_{i}$ satisfy the hypotheses of Theorem 1.7. The problem of determining the diffeomorphism types of such sums now naturally splits into 3 cases, which we address in turn.

3.1. Case 1: $X_{1}$ and $X_{2}$ are rational. Note that, since the $F_{i}$ are tori, we have $\kappa_{X_{1} \#_{F_{1}}=F_{2} X_{2}}^{2}=3 \sigma\left(X_{1} \#_{F_{1}=F_{2}} X_{2}\right)+2 \chi\left(X_{1} \#_{F_{1}}=F_{2} X_{2}\right)=\kappa_{X_{1}}^{2}+\kappa_{X_{2}}^{2}$. Now the rational surfaces have $\kappa_{\mathbb{C} P^{2} \# k \overline{\mathbb{C} P^{2}}}^{2}=9-k$ and $\kappa_{S^{2} \times S^{2}}^{2}=8$, in light of which, in order for $\kappa_{X_{1} \#_{F_{1}}=F_{2} X_{2}}^{2}$ to be zero, the unordered pair $\left\{X_{1}, X_{2}\right\}$ must be either $\left\{\mathbb{C} P^{2} \#\right.$ $\left.k \overline{\mathbb{C} P^{2}}, \mathbb{C} P^{2} \#(18-k) \overline{\mathbb{C} P^{2}}\right\}(0 \leq k \leq 9)$ or $\left\{S^{2} \times S^{2}, \mathbb{C} P^{2} \# 17 \overline{\mathbb{C} P^{2}}\right\}$. Note that since $\left[F_{i}\right]=-\mathrm{PD}\left(\kappa_{X_{i}}\right), F_{i}$ has intersection number 1 with each embedded symplectic (-1)-sphere in $X_{i}$. Choosing an almost complex structure $J_{i}$ on $X_{i}$ generic among those making $F_{i}$ pseudoholomorphic, each homology class in $X_{i}$ that is represented by an embedded symplectic $(-1)$-sphere will be represented by a $J_{i}$-holomorphic $(-1)$-sphere, which will then intersect $F_{i}$ transversely and once. Blowing down such a $(-1)$-sphere will then result in a symplectic manifold $X_{i}^{\prime}$ together with an embedded torus $F_{i}^{\prime}$, with $\left[F_{i}^{\prime}\right]^{2}=\left[F_{i}\right]^{2}+1$.

Now according to Lemma 5.1 of [7] (respectively, Proposition 1.6 of [22]), if $\left(M_{1}, \omega_{1}\right),\left(M_{2}, \omega_{2}\right)$ are symplectic 4-manifolds containing surfaces $\Sigma_{1}, \Sigma_{2}$ of equal genus and area such that $\left[\Sigma_{1}\right]^{2}+\left[\Sigma_{2}\right]^{2}=1$, and if $\left(\widetilde{M}_{i}, \widetilde{\Sigma}_{i}\right)$ denotes the pair consisting of a symplectic 4-manifold and symplectic surface that results from blowing up $M_{i}$ at a point of $\Sigma_{i}$, then $\tilde{M}_{1} \#_{\tilde{\Sigma}_{1}=\Sigma_{2}} M_{2}$ is diffeomorphic (respectively, symplectic deformation equivalent) to $M_{1} \#_{\Sigma_{1}=\tilde{\Sigma}_{2}} \tilde{M}_{2}$.

Applying this to the cases under consideration enables us to replace the pair $\left\{\mathbb{C} P^{2} \# k \overline{\mathbb{C} P^{2}}, \mathbb{C} P^{2} \#(18-k) \overline{\mathbb{C} P^{2}}\right\}(0 \leq k \leq 9)$ by $\left\{\mathbb{C} P^{2} \#(k+1) \overline{\mathbb{C} P^{2}}, \mathbb{C} P^{2} \#\right.$ $\left.(18-k-1) \overline{\mathbb{C} P^{2}}\right\}$ (since if $X_{2}=\mathbb{C} P^{2} \#(18-k)$ where $k \leq 9, X_{2}$ contains $(-1)$ spheres meeting $F_{2}$ transversely once, any one of which may be blown down to give a torus of square one larger in $\mathbb{C} P^{2} \#(18-k-1) \overline{\mathbb{C} P^{2}}$, and we may then apply Gompf's and McDuff-Symington's results mentioned above). Repeatedly "trading blowups" in this fashion enables us to reduce to the case that $k=18-k=9$. 
Similarly, if $X_{1}=S^{2} \times S^{2}$, then $X_{2}=\mathbb{C} P^{2} \# 17 \overline{\mathbb{C} P^{2}}$, so "trading blowups" once reduces us to the case that $X_{1}=\left(S^{2} \times S^{2}\right) \# \overline{\mathbb{C} P^{2}}=\mathbb{C} P^{2} \# 2 \overline{\mathbb{C} P^{2}}$ and $X_{2}=\mathbb{C} P^{2} \# 16 \overline{\mathbb{C} P^{2}}$, and then doing so seven more times reduces to the case that $X_{1}=X_{2}=\mathbb{C} P^{2} \# 9 \overline{\mathbb{C} P^{2}}$.

So we assume for the rest of this subsection that $X_{1}=X_{2}=\mathbb{C} P^{2} \# 9 \overline{\mathbb{C} P^{2}}$. The $X_{i}$ are then both diffeomorphic to the total space of a rational elliptic surface $E(1)$, and the $F_{i}$ are homologous to a fiber of $E(1)$. In fact, we can make a stronger statement about the $F_{i}$; we begin with the following lemma.

Lemma 3.2. Let $B \subset \mathbb{C}^{2}$ be a closed ball around the origin, and let $\phi: B \rightarrow$ $\mathbb{C}^{2}$ be an orientation preserving diffeomorphism onto a closed subset of $\mathbb{C}^{2}$ which maps $\{(w, z) \in B \mid z=0\}$ orientation-preservingly to $\mathbb{C} \times\{0\}$. Then there is a diffeomorphism $\phi^{\prime}: B \rightarrow \phi(B)$ which is isotopic to $\phi$ by an isotopy which is the identity on a neighborhood of $\partial B$, such that $\phi^{\prime}$ maps $\{(w, z) \in B \mid z=0\}$ to $\mathbb{C} \times\{0\}$ and is holomorphic on a neighborhood of the origin.

Proof. With respect to the splitting $\mathbb{R}^{4}=\mathbb{C}^{2}=\mathbb{R}^{2} \times \mathbb{R}^{2}$, the Jacobian $J$ of $\phi$ at the origin has the $2 \times 2$ block form $\left(\begin{array}{cc}A & M \\ 0 & C\end{array}\right)$. The orientation-preserving conditions ensure that $A$ and $J$ both have positive determinant, in light of which $C$ also has positive determinant. Let $\chi: B \rightarrow[0,1]$ be a smooth function which is equal to 1 on a neighborhood of the origin and to 0 on a neighborhood of $\partial B$. Let $V_{1}$ be the vector field on $B \subset \mathbb{R}^{4}$ whose value at $v \in B$ is $\chi(v)\left(\begin{array}{cc}-\log A & 0 \\ 0 & -\log C\end{array}\right) v$; by composing $\phi$ with the time-1 map of $V_{1}$ we reduce to the case where the Jacobian at the origin has form $\left(\begin{array}{cc}I & A^{-1} M \\ 0 & I\end{array}\right)$. Then where $V_{2}(v)=\chi(v)\left(\begin{array}{cc}0 & -A^{-1} M \\ 0 & M\end{array}\right) v$ composing with the time-1 map of $V_{2}$ reduces us to the case that the Jacobian of $\phi$ at the origin is the identity. (Note that both $V_{1}$ and $V_{2}$ are tangent to $\mathbb{C} \times\{0\}$, so the condition that this set be preserved is not disturbed). But in this case there is a constant $C$ (depending on the second derivatives of $\phi)$ such we have $|\phi(w, z)-(w, z)| \leq C\left(|w|^{2}+|z|^{2}\right)$ and $\left|(D \phi)_{(w, z)}-I\right| \leq C\left(|w|^{2}+|z|^{2}\right)^{1 / 2}$. Let $\beta:[0, \infty) \rightarrow[0,1]$ be a smooth, monotone function such that $\beta(r)=0$ for $r \leq 1 / 3, \beta(r)=1$ for $r \geq 1$, and $\beta^{\prime}(r) \leq 2$. Then if $\delta>0$ defining

$$
\phi^{\prime}(w, z)=(w, z)+\beta\left(\left(|w|^{2}+|z|^{2}\right)^{1 / 2} / \delta\right)(\phi(w, z)-(w, z))
$$

yields a smooth map which coincides with $\phi$ outside the ball of radius $\delta$ around the origin, is holomorphic in the ball of radius $\delta / 3$ around the origin, and differs from $\phi$ in $C^{1}$ norm by at most $(3+\delta) C \delta$ and therefore is a diffeomorphism as long as $\delta$ is taken small enough. Also, since $\phi$ preserves $\{z=0\}, \phi^{\prime}$ evidently does as well, and $\phi^{\prime}$ is isotopic to $\phi$ by the isotopy $\phi+t\left(\phi^{\prime}-\phi\right)$.

Lemma 3.3. Let $(M, \omega)$ be a symplectic manifold obtained by blowing up $\left(\mathbb{C} P^{2}, \omega_{F S}\right)$ at nine distinct points, and let $F \subset M$ be an embedded symplectic submanifold Poincaré dual to the anticanonical class $-\kappa_{M}$. Then there exists 
an elliptic fibration $\pi: E(1) \rightarrow S^{2}$ with no multiple fibers and a diffeomorphism $\Phi: M \rightarrow E(1)$ with the property that $\Phi(F)$ is a fiber of $\pi$.

Proof. Let $\tilde{J}_{0}$ be an almost complex structure which makes $F$ pseudoholomorphic. Provided that $\tilde{J}_{0}$ is chosen generically from among such almost complex structures, there are unique, embedded $\tilde{J}_{0}$-holomorphic representatives $E_{1}, \ldots, E_{9}$, respectively, of the homology classes of the nine exceptional divisors of the blowup $M \rightarrow \mathbb{C} P^{2}$. Each of the $E_{i}$ has a tubular neighborhood $N_{i}$ modeled (symplectically) on a neighborhood of the zero section of the holomorphic line bundle $\mathcal{O}(-1)$ over $\mathbb{C} P^{1}$; we may shrink the $N_{i}$ if necessary to make them pairwise disjoint. Now, for each $i$, the $\tilde{J}_{0}$-holomorphic curves $F$ and $E_{i}$ have homological intersection number 1, so they meet transversely, positively, and in just one point. Hence as in Lemma 2.3 of [7], we may isotope $F$ rel $M \backslash \cup N_{i}$ by symplectomorphisms to a surface $F^{\prime}$ such that, for some smaller neighborhoods $N_{i}^{\prime}$ of $E_{i}, F^{\prime} \cap N_{i}^{\prime}$ coincides with a fiber of the bundle projection $\mathcal{O}(-1) \rightarrow E_{i}$. Further, during this isotopy, we can change the almost complex structure $\tilde{J}_{0}$ to an almost complex structure $\tilde{J}$ which agrees with $\tilde{J}_{0}$ outside $N_{i}$, makes $F^{\prime}$ holomorphic, and coincides on $N_{i}^{\prime}$ with the standard integrable complex structure on the tautological line bundle $\mathcal{O}(-1)$.

We now symplectically blow down the $E_{i}$ (see Section 7.1 of [21] for a detailed description of this process); doing so amounts to symplectically identifying an annular neighborhood $L_{i}=D\left(\delta_{1, i}\right) \backslash D\left(\delta_{2, i}\right) \subset N_{i}^{\prime} \subset \mathcal{O}(-1)$ of the zero section $E_{i}$ with a spherical shell $B\left(r_{1, i}\right) \backslash B\left(r_{2, i}\right)$ in $\mathbb{C}^{2}$, and filling in this shell by a standard ball (centered at a point $p_{i}$ with radius $r_{2, i}$ ) to replace the $\delta_{2, i}$-neighborhood of $E_{i}$. This process is compatible with the complex blowdown in the sense that the annulus fibers of $L_{i}$ are taken to annuli in the complex lines that they correspond to under the identification of $\mathcal{O}(-1)$ with the tautological line bundle of $\mathbb{C}^{2}$, so in the blowdown these annuli may be filled in to form discs by adding in the disc of radius $r_{2, i}$ in the corresponding complex line. So since $F^{\prime}$ meets each $L_{i}$ in one of these annulus fibers, filling these annuli in as above gives rise to a compact symplectic surface $S$ whose intersection with each $B\left(r_{1, i}\right)$ coincides with a complex line.

As a result of all this, blowing down the $E_{i}$ results in $\mathbb{C} P^{2}$ equipped with an almost complex structure $J$ which is integrable near each of the points $p_{i}$ (and coincides with $\tilde{J}$ outside the blow-up neighborhoods), a $J$-holomorphic curve $S \subset \mathbb{C} P^{2}$ which coincides with $F^{\prime}$ outside the blow-up neighborhoods, and a symplectic form $\omega^{\prime}$ (induced by $\omega$ and the blowdown procedure) which is compatible with $J . \omega^{\prime}$ is easily seen to be cohomologous to the Fubini-Study form (for instance, the proper transform of the hyperplane class under the original blowups that were done to obtain $M$ from $\mathbb{C} P^{2}$ has a nonvanishing Gromov-Witten invariant, and so may be represented by a symplectic surface $\widetilde{H}$ which we can arrange to miss the $L_{i}$ and so gives rise to a symplectic surface in $\left(\mathbb{C} P^{2}, \omega^{\prime}\right)$ with the same area as $\widetilde{H}$ has in $\left.(M, \omega)\right)$. Hence by a result of [10] $\omega^{\prime}$ is symplectomorphic to the Fubini-Study form. Also, for 
a similar reason as above, $S \subset \mathbb{C} P^{2}$ has degree 3. So by Theorem 3 of [25], $S$ is symplectically isotopic to some complex cubic curve $C$. The isotopy extension theorem then ensures that there is an ambient isotopy $\phi_{t}: \mathbb{C} P^{2} \rightarrow \mathbb{C} P^{2}$ such that $\phi_{1}(S)=C$. Our intention now is to modify $\phi_{1}$ to some other diffeomorphism $\phi_{2}$ so that (i) $\phi_{2}(S)=C$, (ii) $\mathbb{C} P^{2}$ carries an elliptic pencil with fiber $C$ and base locus $\left\{\phi_{2}\left(p_{1}\right), \ldots, \phi_{2}\left(p_{9}\right)\right\}$, and (iii) $\phi_{2}$ lifts to a diffeomorphism of the appropriate blowups which takes $F^{\prime}$ to the proper transform of $C$.

To achieve this, first note that since the sheaf of sections of the holomorphic normal bundle to $C$ which vanish at the eight points $\phi_{1}\left(p_{1}\right), \ldots, \phi_{1}\left(p_{8}\right)$ has degree 1, it admits global nonvanishing sections by Riemann-Roch; perturbing $\phi_{1}$ by composing with a small diffeomorphism which preserves $C$ we can arrange that $\phi_{1}\left(p_{1}\right), \ldots, \phi_{1}\left(p_{8}\right)$ are generic in the sense that none of these sections vanishes to order 2. Such a section results in another smooth cubic $C^{\prime}$ which meets $C$ transversely at the eight points $\phi_{1}\left(p_{1}\right), \ldots, \phi_{1}\left(p_{8}\right)$, and also at some other point $q_{9}$. Now let $v$ be a vector field on $C$ which vanishes on neighborhoods of the $\phi_{1}\left(p_{i}\right)$ for $1 \leq i \leq 8$ and whose time-1 flow maps $\phi_{1}\left(p_{9}\right)$ to $q_{9}$. Using a partition of unity subordinate to a set of local trivializations of the normal bundle to $C$ and a bump function supported on a tubular neighborhood of $C$, extend $v$ to a vector field $V$ on $\mathbb{C} P^{2}$ whose restriction to $C$ is $v$; let $\psi$ be the time- 1 flow of $V$, and let $\phi_{0}=\psi \circ \phi_{1}$. Then $\phi_{0}: \mathbb{C} P^{2} \rightarrow \mathbb{C} P^{2}$ is a diffeomorphism which maps $S$ to $C$ and $p_{1}, \ldots, p_{9} \in S$ to the 9 intersection points between $C$ and another smooth cubic $C^{\prime}$.

Now $\phi_{0}$ will not lift to a diffeomorphism on blowups, because it is not holomorphic near the points being blown up and so does not map complex lines to complex lines. However, around each $p_{i}$ there are complex coordinates $(w, z)$ in which $S$ is given by $\{z=0\}$ and likewise near each $\phi_{0}\left(p_{i}\right)$ there are complex coordinates in which $C$ is given by $\{z=0\}$. Hence, in terms of these local holomorphic coordinates, $\phi_{0}$ is given in these neighborhoods by a diffeomorphism which satisfies the hypothesis of Lemma 3.2. So $\phi_{0}$ may be modified by an isotopy supported in the union of these neighborhoods to an orientation-preserving diffeomorphism $\phi_{2}: \mathbb{C} P^{2} \rightarrow \mathbb{C} P^{2}$ which maps $S$ to $C$ and is holomorphic on (smaller) neighborhoods of $p_{1}, \ldots, p_{9}$. Consequently, if $Y$ is the (complex) blowup of $\left(\mathbb{C} P^{2}, J\right)$ at the 9 points $p_{1}, \ldots, p_{9}$ (which makes sense because $J$ is integrable near the $p_{i}$ ), and if $E(1)$ is the complex blowup of $\left(\mathbb{C} P^{2}, J_{\text {std }}\right)$ at $\phi_{2}\left(p_{1}\right), \ldots, \phi_{2}\left(p_{9}\right), \phi_{2}$ lifts to a diffeomorphism $Y \rightarrow$ $E(1)$ taking the proper transform of $S$ to the proper transform of $C$. If $f$ and $f^{\prime}$ are homogeneous cubic polynomials with vanishing loci $C$ and $C^{\prime}$ respectively, the vanishing loci $C_{[\lambda: \mu]}$ of $\lambda f+\mu f^{\prime}\left([\lambda: \mu] \in \mathbb{C} P^{1}\right)$ provide an elliptic pencil on $\mathbb{C} P^{2}$ with base locus $\left\{\phi\left(p_{1}\right), \ldots, \phi\left(p_{9}\right)\right\}$. Blowing up the base locus to form $E(1)$ thus gives an elliptic fibration with the proper transform of $C$ as a fiber.

To compare $Y$ to $X$, note that in order to put a symplectic form on the complex blowup $Y$ in such a way that the map $\phi_{2}$ lifts to $Y$ we need to cut out balls $B_{i}^{\prime}$ around $p_{i}$ that are smaller than the balls $B\left(r_{2, i}\right)$ that were created by the blowdown 
$X \rightarrow \mathbb{C} P^{2}$. $X$ and $Y$ hence cannot be symplectomorphic. However, there is an obvious diffeomorphism between the blowups corresponding to balls of different size which simply changes the radius of the disc bundle involved; in particular this diffeomorphism has a restriction to the neighborhood of the exceptional sphere which preserves the fibers of the normal bundle $\mathcal{O}(-1)$. Recalling that $F^{\prime}$ coincided with a fiber of the normal bundle on the neighborhood $N_{i}^{\prime}$ it then follows that the natural diffeomorphism $X \rightarrow Y$ takes $F^{\prime}$ to the proper transform of $S$ in $Y$.

We hence have a diffeomorphism $X \rightarrow E(1)$ which takes $F^{\prime}$ to the proper transform of $C$; precomposing this with a symplectomorphism of $X$ which isotopes $F$ to $F^{\prime}$ gives us the promised map $\Phi: X \rightarrow E(1)$ taking $F$ to a fiber of an elliptic fibration.

Corollary 3.4. If the symplectic sum $Z$ of two rational surfaces along a positive genus surface is a minimal symplectic four-manifold of Kodaira dimension zero, then $Z$ is diffeomorphic to the $K 3$ surface.

Proof. Denote the two summands by $X_{i}(i=1,2)$ and the surfaces in question by $F_{i}$. We have shown that we may assume that $X_{i}=\mathbb{C} P^{2} \# 9 \overline{\mathbb{C} P^{2}}$ and that the $F_{i}$ are tori Poincaré dual to $-\mathrm{PD}\left(\kappa_{X_{i}}\right)$. Hence by Lemma 3.3, we have $X_{i} \backslash v F_{i} \cong E(1) \backslash v F$ where $E(1)$ is the total space of a rational elliptic fibration having fiber $F$ (and where “@” denotes diffeomorphism). Hence

$$
Z \cong(E(1) \backslash v F) \cup_{\Phi}(E(1) \backslash v F)
$$

for some orientation-reversing diffeomorphism $\Phi$ of the boundary $\partial(E(1) \backslash v F)=$ $T^{3}$. But according to Proposition 1 of Appendice 4 of [11], every orientation preserving diffeomorphism of $\partial(E(1) \backslash v F)$ extends to $E(1) \backslash \nu F$, so the diffeomorphism type of $(E(1) \backslash v F) \cup_{\Phi}(E(1) \backslash v F)$ is independent of $\Phi$. So since one choice of $\Phi$ (namely the one corresponding to taking the standard fiber sum of $E(1)$ with itself) gives rise to the $K 3$ surface, $Z$ is evidently diffeomorphic to the $K 3$ surface independently of $\Phi$.

3.2. Case 2: $X_{1}$ is rational and $X_{2}$ is irrational and ruled. Assume that $X_{2}$ is a ruled surface over a curve $C$ of positive genus; we'll show shortly that $C$ is a torus in the cases of interest. Then $X_{2}$ is symplectomorphic either to the nontrivial $S^{2}$-bundle over $C$, which we denote $S^{2} \tilde{\times} C$, or else $X_{2}=\left(S^{2} \times C\right) \# k \overline{\mathbb{C} P^{2}}$ for some $k \geq 0$. Suppose that $X_{1}$ is rational and $F_{i} \subset X_{i}(i=1,2)$ are embedded symplectic submanifolds with the property that the smoothly nontrivial symplectic sum $X_{1} \#_{F_{1}}=F_{2} X_{2}$ is minimal and of Kodaira dimension zero. Then Theorem 3.1 shows that the $F_{i}$ are tori Poincaré dual to $-\kappa_{F_{i}}$. In particular, if $X_{2}$ is nonminimal and $J_{2}$ is an almost complex structure preserving $T F_{2}$, then each member of a maximal disjoint collection of embedded $J_{2}$-holomorphic (-1)-spheres meets $F_{2}$ transversely 
and once; hence the results of [7] and [22] alluded to in the previous subsection show that, up to deformation equivalence, the symplectic sum is left unchanged if we simultaneously blow down each member of this maximal collection and blow up $X_{1}$ at a corresponding number of points on $F_{1}$. This reduces us to the case that $X_{2}$ is minimal, and so is either $S^{2} \times C$ or $S^{2} \tilde{\times} C$.

Lemma 3.5. Let $\pi: E \rightarrow C$ be an $S^{2}$-bundle over a positive-genus surface $C$ with symplectic form $\omega \in \Omega^{2}(E)$, and let $\Sigma \subset E$ be an embedded, connected, symplectic representative of $-\mathrm{PD}\left(\kappa_{E}\right) \in H_{2}(E ; \mathbb{Z})$, with tubular neighborhood $v \Sigma$. Then $C$ is a torus and there is another bundle map $\pi^{\prime}: E \rightarrow C$ whose fibers are symplectic spheres such that $\left.\pi^{\prime}\right|_{E \backslash \nu \Sigma}$ defines a fiber bundle with fibers diffeomorphic to the annulus $S^{1} \times I$.

Proof. First, note that if $J$ is an almost complex structure compatible with $\omega$ with respect to which $\Sigma$ is pseudoholomorphic, and if $\mathcal{M}$ is the moduli space of unparametrized pseudoholomorphic spheres representing the class of the fiber of $\pi$, then results of [19] show that the map $\pi^{\prime}: E \rightarrow \mathcal{M}$ which takes $e \in E$ to the point of $\mathcal{M}$ representing the unique $J$-holomorphic representative of the fiber class on which $e$ lies is an $S^{2}$-bundle with fibers homologous to the fibers of $\pi$; fundamental group considerations then imply that $\mathcal{M}$ has the same genus as $C$. By construction the fibers of $\pi^{\prime}$ are $J$-holomorphic and hence symplectic.

We now claim that $\Sigma$ is isotopic to some surface $\Sigma^{\prime} \subset E$ such that there is an almost complex structure $J^{\prime}$ which makes $\Sigma^{\prime}$ pseudoholomorphic and with respect to which $\pi^{\prime}: E \rightarrow \mathcal{M}$ is a pseudoholomorphic map (with respect to some almost complex structure on $\mathcal{M}$ ). Indeed, as in the proof of Lemma 3.2 of [16], using the parametrized Riemann mapping theorem we can find complex coordinates $(z, w)$ on a suitable open set $U_{i}$ of $E$ centered around any critical point $p_{i}$ of $\left.\pi^{\prime}\right|_{\Sigma}$ in terms of which the projection $\pi^{\prime}: E \rightarrow \mathcal{M}$ is given by $(z, w) \mapsto w$ and $\partial_{\bar{z}}$ lies in the $J$ antiholomorphic tangent space $T_{J}^{0,1}$. The intersection of $\Sigma$ with this neighborhood will then be given by $\Sigma \cap U_{i}=\left\{w=g_{i}(z)\right\}$ where $g_{i}(z)=c_{i} z^{k_{i}}+O\left(|z|^{k_{i}+1}\right)$ and $c_{i} \neq 0$; that $\left.\pi^{\prime}\right|_{\Sigma}$ has a critical point at $(0,0)$ amounts to the statement that $k_{i}>1$. Note that since $\Sigma$ has intersection number 2 with the fibers of $\pi^{\prime}$ (as $[\Sigma]=-\kappa_{E}$ and the fibers are square-zero spheres), we in fact have $k_{i}=2$, and moreover there can only be one critical point of $\left.\pi^{\prime}\right|_{\Sigma}$ in any given fiber of $\pi^{\prime}$; accordingly we can and do choose the $U_{i}$ so that the $\pi^{\prime}\left(U_{i}\right)$ are disjoint as $i$ varies. We can then use a cutoff function supported in $U_{i}$ and equal to 1 on some smaller neighborhood $U_{i}^{\prime}$ of the critical point $p_{i}$ to isotope $\Sigma$ rel $E \backslash U_{i}$ to some new surface $\Sigma^{\prime}$ whose intersection with $U_{i}^{\prime}$ is given by $\Sigma^{\prime} \cap U_{i}^{\prime}=\left\{w=c_{i} z^{k_{i}}\right\}$; further, using the same cutoff, we can isotope $J$ rel $E \backslash U_{i}$ to a new almost complex structure $J^{\prime}$ which coincides with the standard integrable complex structure with holomorphic coordinates $(z, w)$ on $U_{i}^{\prime}$, and with respect to which both $\Sigma^{\prime}$ and the fibers of $\pi^{\prime}$ are $J^{\prime}$-holomorphic. Repeating this 
near every critical point of $\left.\pi^{\prime}\right|_{\Sigma}, \Sigma^{\prime}$ and the fibers of $\pi^{\prime}$ are now $J^{\prime}$-holomorphic and $\pi^{\prime}: E \rightarrow \mathcal{M}$ restricts to a pseudoholomorphic map on a neighborhood of $\operatorname{Crit}\left(\pi^{\prime} \mid \Sigma^{\prime}\right)$ with respect to $J^{\prime}$ on $E$ and a suitable complex structure on $\cup_{i} \pi^{\prime}\left(U_{i}^{\prime}\right) \subset \mathcal{M}$. But then extending this complex structure to all of $\mathcal{M}$, since $\left.\pi^{\prime}\right|_{\Sigma \backslash \cup_{i} U_{i}^{\prime}}$ is an unbranched cover a simple patching argument may be used to further modify $J^{\prime}$ so that it continues to make $\Sigma^{\prime}$ pseudoholomorphic and now also makes the whole projection $\pi^{\prime}: E \rightarrow \mathcal{M}$ pseudoholomorphic. This proves the claim at the start of this paragraph.

But now $\left.\pi^{\prime}\right|_{\Sigma^{\prime}}: \Sigma^{\prime} \rightarrow \mathcal{M}$ is a holomorphic map from a torus to $\mathcal{M}$ with degree 2; we know that $\mathcal{M}$ has the same positive genus as $C$, so it follows that that genus is one. Hurwitz's formula then implies that $\left.\pi^{\prime}\right|_{\Sigma^{\prime}}$ has no critical points; since the critical points of $\left.\pi^{\prime}\right|_{\Sigma^{\prime}}$ were constructed to be just the same as those of $\left.\pi^{\prime}\right|_{\Sigma}$ it then follows that $\left.\pi^{\prime}\right|_{\Sigma}$ has no critical points. Thus $\Sigma$ meets every fiber of $\pi^{\prime}$ transversely, and hence exactly twice by the positivity of intersections of $J$-holomorphic curves. Hence

$$
\left.\pi^{\prime}\right|_{E \backslash \nu \Sigma}: E \backslash \nu \Sigma \rightarrow T^{2}
$$

is a fibration with fiber given by the complement of two discs in $S^{2}$, i.e., by $S^{1} \times I$.

Consequently, in all cases of interest, we have $X_{2}=S^{2} \times T^{2}$ or $X_{2}=S^{2} \tilde{\times} T^{2}$; these both have $c_{1}^{2}=0$, and so if $X_{1} \#_{F_{1}=F_{2}} X_{2}$ is to be minimal of Kodaira dimension zero then $c_{1}^{2}\left(X_{1}\right)=0$. Since the only rational surface with $c_{1}^{2}=0$ is $\mathbb{C} P^{2} \# 9 \overline{\mathbb{C} P^{2}}$, evidently $X_{1}=\mathbb{C} P^{2} \# 9 \overline{\mathbb{C} P^{2}}$.

The above lemma makes the diffeomorphism classification of annulus bundles over $T^{2}$ relevant to us; specifically we are interested in those annulus bundles with orientable total space and having just one boundary component. Identify $S^{1} \times I$ with $A=\overline{D(2)} \backslash D(1 / 2) \subset \mathbb{C}$. Any annulus bundle over $T^{2}$ is isomorphic to one of the form $M\left(f, g ;\left\{h_{t}\right\}\right)$ where $f, g \in \pi_{0}(\operatorname{Diff}(A))$ commute, $\left\{h_{t}\right\}_{t \in S^{1}} \in \pi_{1}(\operatorname{Diff}(A))$,

$$
M(f, g ;\{1\})=\frac{R^{2} \times A}{(x+1, y, z) \sim(x, y, f(z)),(x, y+1, z) \sim(x, y, g(z))}
$$

and $M\left(f, g ;\left\{h_{t}\right\}\right)$ is obtained from $M(f, g ;\{1\})$ by removing a trivial neighborhood $D^{2} \times A$ from $M(f, g ;\{1\})$ and gluing it back by the map

$$
\begin{aligned}
\partial D^{2} \times A & \rightarrow \partial D^{2} \times A, \\
(t, z) & \rightarrow\left(t, h_{t}(z)\right) .
\end{aligned}
$$

Since changing the choice of basis $\{u, v\}$ of $H_{1}\left(T^{2} ; \mathbb{Z}\right)$ to, respectively, $\{u+v, v\}$ or $\{v, u\}$ corresponds to replacing $(f, g)$ by $(f \circ g, g)$ or $(g, f)$, we can assume that $f$ maps each respective boundary component of $A$ to itself (if $f$ does not initially, then either $g$ or $f \circ g$ does). Now $\pi_{0}(\operatorname{Diff}(A))=\mathbb{Z}_{2} \oplus \mathbb{Z}_{2}$, with generators given by $z \mapsto z^{-1}$ and $z \mapsto \bar{z}$. 
We are interested in orientable annulus bundles over $T^{2}$ having just one boundary component. The orientability condition restricts us to the case that the monodromies $f$ and $g$ preserve the orientation of $A=\overline{D(2)} \backslash D(1 / 2) . f$ is assumed to map each boundary component to itself, so this forces $f$ to be isotopic to the identity. But then in order for the bundle to have just one boundary component $g$ must swap the boundary components of $A$, forcing $g$ to be isotopic to $z \mapsto z^{-1}$.

Now as explained after the statement of Theorem 2.3 in [28], it follows from a theorem of Smale that the identity component of $\operatorname{Diff}(A)$ retracts to $S^{1}$ (and indeed the map $\operatorname{Diff}_{0}(A) \rightarrow \operatorname{Diff}_{0}\left(S^{1}\right)$ given by restriction to one boundary component is a homotopy equivalence), so $\pi_{1}(\operatorname{Diff}(A))$ is generated by the loop of diffeomorphisms $r_{t}: A \rightarrow A$ where for $t \in S^{1} r_{t}$ is given by rotation through the angle $t$. Thus, any orientable annulus bundle over $T^{2}$ with one boundary component has form

$$
Y_{n}=M\left(I, z \mapsto z^{-1} ;\left\{r_{t}^{n}\right\}\right)
$$

In fact, arguing exactly as in Lemma 7 of Section 8 of [23], where $l, m \in \pi_{1}\left(\partial D^{2} \times A\right)$ are, respectively, the generators of the images of the inclusion-induced maps $\pi_{1}(A) \rightarrow$ $\pi_{1}\left(\partial D^{2} \times A\right)$ and $\pi_{1}\left(\partial D^{2}\right) \rightarrow \pi_{1}\left(\partial D^{2} \times A\right)$, one finds that for any $n \in \mathbb{Z}$ there is a fiber preserving diffeomorphism $Y_{0} \backslash D^{2} \times A \rightarrow Y_{0} \backslash D^{2} \times A$ whose restriction to the boundary $\partial D^{2} \times A$ takes a representative of $m$ to a representative of $m+2 n l$ (an explicit formula for such a diffeomorphism may easily be found by adapting the proof of Proposition 2(3) of [24] to the case where the fibers of the bundles involved are annuli rather than tori). Thus, every orientable annulus bundle over the torus having just one boundary component is isomorphic as a smooth fiber bundle to either $Y_{0}$ or $Y_{1}$.

By definition, we have

$$
Y_{0}=S^{1} \times \frac{\mathbb{R} \times A}{(x+1, z) \sim\left(x, z^{-1}\right)}
$$

Meanwhile, we see easily that

$$
Y_{1}=\frac{\mathbb{R} \times S^{1} \times A}{\left(x+1, e^{i \theta}, z\right) \sim\left(x, e^{i \theta}, e^{i \theta} z^{-1}\right)},
$$

since the right hand side above obviously admits the structure of an annulus bundle and so by our earlier remarks is isomorphic either to $Y_{0}$ or to $Y_{1}$; computation of the fundamental group then shows that it is distinct from $Y_{0}$.

Lemma 3.6. Let $\pi: E \rightarrow T^{2}$ be an $S^{2}$-bundle with symplectic form $\omega \in \Omega^{2}(E)$, and let $\Sigma \subset E$ be an embedded, connected, symplectic representative of $-\mathrm{PD}\left(\kappa_{E}\right) \in$ $\mathrm{H}_{2}(E ; \mathbb{Z})$, with tubular neighborhood $v \Sigma$. Then $E \backslash v \Sigma$ is diffeomorphic to $Y_{0}$ if and only if $E$ is symplectomorphic to $S^{2} \times T^{2}$ (with some split symplectic form). 
Proof. By Lemma 3.5, possibly after redefining $\pi$ we may assume that $\pi$ has symplectic fibers and that $\left.\pi\right|_{\Sigma \backslash \nu \Sigma}$ defines an annulus bundle over $T^{2}$.

For the forward implication, simply note that the annulus bundle $Y_{0}$ admits a section $(\theta, x) \mapsto[\theta, x, 1]$; if $E \backslash v \Sigma$ is diffeomorphic to $Y_{0}$ this section includes into $E$ as a torus which intersects the fibers of $E \rightarrow T^{2}$ once transversely and which misses $\Sigma$. Now the total space of $E$ is, by results of [19], symplectomorphic by a fiber-preserving map to the projectivization a complex line bundle over $T^{2}$ of degree either 0 or 1 ; however, in the projectivization of a line bundle of degree 1 over the torus there are no homology classes having intersection number 1 with the fibers and 0 with the anticanonical class. Hence $E$ must be the projectivization of the trivial complex line bundle over $T^{2}$, i.e. $S^{2} \times T^{2}$.

Conversely, suppose that $E$ is symplectomorphic to $S^{2} \times T^{2}$. As in Lemma 3.5, we can assume that $\Sigma$ meets each fiber of $\pi: E \rightarrow T^{2}$ transversely twice. Now let $p: M \rightarrow S^{2}$ be a nontrivial $S^{2}$-bundle over $S^{2}$, and let $F \subset M$ be the disjoint union of a section of square -1 and a section of square 1 of $p$, each of which is symplectic with respect to some symplectic form on $F$ which restricts nondegenerately to the fibers of $\pi$. Then by the pairwise sum construction in [7], the fiber sum $E^{\prime}$ of $M$ and $E$ carries a symplectic form and admits a symplectic torus $\Sigma^{\prime}$ obtained by gluing $\Sigma$ to the section of square -1 in $F$ at one of its intersection points with the fiber and to the section of square 1 in $F$ at the other. Now the induced $S^{2}$-fibration $\pi^{\prime}: E^{\prime} \rightarrow T^{2}$ on the fiber sum is easily seen to admit sections of odd square (glue a section of even square in $E$ to a section of odd square in $M$ ), so $E^{\prime}$ is diffeomorphic to the nontrivial $S^{2}$-bundle over $T^{2}$. Hence by the previous paragraph $E^{\prime} \backslash \nu \Sigma^{\prime}$ is not diffeomorphic to $Y_{0}$, so it is diffeomorphic to $Y_{1}$.

Now $M$ is diffeomorphic to $\mathbb{C} P^{2} \# \overline{\mathbb{C} P^{2}}$; the complement $M \backslash v F$ of a neighborhood of the disjoint union $F$ of a section of square 1 and a section of square -1 is then diffeomorphic to the complement of a neighborhood of the union of a point and a line in $\mathbb{C} P^{2}$, i.e. to a region $\left\{\left.(z, w) \in \mathbb{C}^{2}|r \leq| z\right|^{2}+|w|^{2} \leq R\right\}$ in $\mathbb{C}^{2}$. In these terms, $\left.\pi\right|_{M \backslash v F}: M \backslash v F \rightarrow \mathbb{C} P^{1}$ is the Hopf map $(z, w) \mapsto[z: w]$. This shows that the annulus fibration $\left.p\right|_{M \backslash \nu F}: M \backslash v F \rightarrow S^{2}$ is obtained from the trivial annulus fibration over $S^{2}$ by removing the neighborhood of a fiber and regluing it by the diffeomorphism $\left(e^{i \theta}, z\right) \mapsto\left(e^{i \theta}, e^{i \theta} z\right)$ of $\partial D^{2} \times A$.

But the annulus fibration $\pi^{\prime}: E^{\prime} \backslash \nu \Sigma^{\prime} \rightarrow T^{2}$ is obtained by taking the fiber sum of $\pi: E \backslash \nu \Sigma \rightarrow T^{2}$ with $p: M \backslash \nu F \rightarrow S^{2}$, so this implies that $\pi^{\prime}: E^{\prime} \backslash \nu \Sigma^{\prime} \rightarrow T^{2}$ may be constructed from $\pi: E \backslash \nu \Sigma \rightarrow T^{2}$ by removing the neighborhood of a fiber and regluing it by the diffeomorphism $\left(e^{i \theta}, z\right) \mapsto\left(e^{i \theta}, e^{i \theta} z\right)$ of $\partial D^{2} \times A$. Now performing this operation on the annulus bundle $Y_{0}$ yields $Y_{1}$, while performing it on $Y_{1}$ yields $Y_{2} \cong Y_{0}$. So since we have already established that $E^{\prime} \backslash \nu \Sigma^{\prime}$ is diffeomorphic to $Y_{1}$, it must be that $E \backslash \nu \Sigma$ is diffeomorphic to $Y_{0}$. 
Theorem 3.7. Let $X$ be an $S^{2}$-bundle over $T^{2}$, let $F \subset X$ be an embedded symplectic representative of $-\mathrm{PD}\left(\kappa_{X}\right)$, and let $F^{\prime} \subset E(1)$ be an embedded symplectic representative of $-\mathrm{PD}\left(\kappa_{E(1)}\right)$. Then the symplectic sum $E(1) \#_{F^{\prime}=F} X$ is diffeomorphic to the Enriques surface.

Proof. First, we notice that we can reduce to the case that $X$ is symplectomorphic to $S^{2} \times T^{2}$ (with some split symplectic form). Indeed, if $X$ is instead diffeomorphic to the nontrivial $S^{2}$-bundle over $T^{2}$, we shall twice apply the result of [7] and [22] which allows us to "trade blowups" as discussed in Case 1. First, if $\left(M_{1}, F_{1}\right)$ is the result of blowing down a $(-1)$-sphere passing once positively and transversely through $F^{\prime}$ (to find such a sphere, use an almost complex structure preserving $T F^{\prime}$ to evaluate the Gromov-Witten invariant of one of the classes of the exceptional spheres of $\left.E(1)=\mathbb{C} P^{2} \# 9 \overline{\mathbb{C} P^{2}}\right)$ and if $\left(M_{2}, F_{2}\right)=(X, F)$, we see that $E(1) \#_{F^{\prime}=F} X$ is deformation equivalent to $M_{1} \#_{F_{1}=\widetilde{F}_{2}} \widetilde{M}_{2}$. Now the ruling $X \rightarrow T^{2}$ induces a genus0 (not relatively minimal) Lefschetz fibration $\pi: \tilde{M}_{2} \rightarrow T^{2}$ each of whose fibers meets the symplectic square- $(-1)$ torus $\widetilde{F}_{2}$ twice; $\pi$ has just one singular fiber, whose components $C_{1}$ and $C_{2}$ are two embedded (-1)-spheres (one of which, say $C_{1}$, is the exceptional sphere of the blowup, and the other of which is the proper transform of the fiber of $X \rightarrow S^{2}$ that passes through the blown-up point), each of which intersects $\widetilde{F}_{2}$ once. Now blowing down $C_{2}$ produces a manifold symplectomorphic to $S^{2} \times T^{2}$, and $\widetilde{F}_{2} \subset M_{2}$ is isotopic to the proper transform of a symplectic representative $F^{\prime \prime}$ of $-\mathrm{PD}\left(\kappa_{S^{2} \times T^{2}}\right)$. Hence $M_{1} \#_{F_{1}=\widetilde{F}_{2}} \widetilde{M}_{2}$ is in turn deformation equivalent to $\tilde{M}_{1} \#_{\widetilde{F}_{1}=F^{\prime \prime}}\left(S^{2} \times T^{2}\right)$. Since $\left(\tilde{M}_{1}, \widetilde{F}_{1}\right)$ is obtained by first blowing down a sphere passing once positively and transversely through $F^{\prime} \subset E(1)$ and then blowing up a point on the image of $F^{\prime}$ under the blowdown, it follows that $\tilde{M}_{1}$ is deformation equivalent to $E(1)$ and $\widetilde{F}_{1}$ represents $-\operatorname{PD}\left(\kappa_{\tilde{M}_{1}}\right)$. This allows us to hereinafter assume that $X=S^{2} \times T^{2}$.

By Lemma 3.3, $E(1) \backslash v F^{\prime}$ is diffeomorphic to the manifold with boundary $N$ obtained by deleting a neighborhood of a regular fiber of an elliptic fibration on $E(1)$, while $X \backslash v F$ is, by Lemma 3.6 and our reduction to the case that $X=S^{2} \times T^{2}$, diffeomorphic to $Y_{0}$.

So the symplectic sum in question is diffeomorphic to

$$
X_{0}=N \cup_{\partial} Y_{0} ;
$$

note that since by Proposition 1 of Appendice 4 of [11] every orientation preserving diffeomorphism of $\partial N$ extends to $N$, the diffeomorphism type of $X_{0}$ is determined independently of the boundary gluing maps.

We claim now that $X_{0}$ is diffeomorphic to the Enriques surface. In fact, this is essentially a remark on p. 50 of [11]; for a direct proof, recall that $Y_{0}=S^{1} \times Z_{0}$ where $Z_{0}=\mathbb{R} \times A /(x+1, z) \sim\left(x, z^{-1}\right)$. Now projecting $Z_{0}$ onto its second factor gives $Z_{0}$ the structure of a Seifert fibration over $D^{2}$ with two multiple 
fibers each having multiplicity 2 ; hence $Y_{0}=S^{1} \times Z_{0}$ is the result of performing two multiplicity two logarithmic transformations on the trivial elliptic fibration $T^{2} \times D^{2}$. Thus $X_{0}=N \cup_{\partial} Y_{0}$ is obtained from $E(1)$ by deleting a neighborhood of a smooth fiber and replacing that neighborhood with the result of two multiplicity two logarithmic transformations on $T^{2} \times D^{2}$, i.e., $X_{0}$ is obtained from $E(1)$ by performing two multiplicity-two logarithmic transformations. But this is precisely the definition of the Enriques surface.

3.3. Case 3: $X_{1}$ and $X_{2}$ are irrational and ruled. Since a $k$-fold blowup of an $S^{2}$-bundle over a surface of genus $h$ has $c_{1}^{2}=8-8 h-k$, in order for the symplectic sum of irrational ruled surfaces $X_{1}$ and $X_{2}$ along a torus to have $c_{1}^{2}=0$, both $X_{1}$ and $X_{2}$ must be $S^{2}$-bundles over $T^{2}$. By Theorem 3.1, the surfaces $F_{i}$ are embedded symplectic tori representing $-\mathrm{PD}\left(\kappa_{X_{i}}\right)$. As in the proof of Lemma 3.5, results of [19] imply that there are projections $\pi_{i}: X_{i} \rightarrow T^{2}$ such that $\left.\pi_{i}\right|_{F_{i}}$ is an unramified double cover of $T^{2}$ by $F_{i}$; the deck transformation of this cover is then a free orientationpreserving involution $\tau_{i}: F_{i} \rightarrow F_{i}$. By considering these involutions, we shall realize any symplectic sum of the $X_{i}$ along the $F_{i}$ as the total space of some torus bundle over $T^{2}$.

Lemma 3.8. Let $\tau_{1}, \tau_{2}: T^{2} \rightarrow T^{2}$ be free orientation-preserving involutions, and let $\phi: T^{2} \rightarrow T^{2}$ be any diffeomorphism. Then $\phi$ is isotopic to a diffeomorphism $\phi^{\prime}: T^{2} \rightarrow T^{2}$ with the property that

$$
\phi^{\prime-1} \circ \tau_{2} \circ \phi^{\prime} \circ \tau_{1} \text { is either the identity or a free involution. }
$$

Proof. First of all, note that any two free orientation-preserving involutions $\tau$ and $\tau^{\prime}$ from $T^{2}$ to $T^{2}$ are conjugate. Indeed, letting $E$ be the quotient of $T^{2}$ by $\tau, E^{\prime}$ the quotient of $T^{2}$ by $\tau^{\prime}$, and $\pi: T^{2} \rightarrow E, \pi^{\prime}: T^{2} \rightarrow E^{\prime}$ the projections, $E$ and $E^{\prime}$ are both tori, so that there exists a diffeomorphism $\psi: E \rightarrow E^{\prime}$. The images of $\pi_{1}\left(T^{2}\right)$ in $\pi_{1}\left(E^{\prime}\right)$ by $\pi^{\prime}$ and $\psi \circ \pi$ are both index 2 lattices in $\pi_{1}\left(E^{\prime}\right) \cong \mathbb{Z}^{2}$, so there is an element $A$ of $\operatorname{SL}(2 ; \mathbb{Z})$ taking one to the other; hence by composing $\psi$ with a diffeomorphism of $E^{\prime}$ that induces $A$ on $\pi_{1}$ we can assume that the maps induced on $\pi_{1}$ by $\pi^{\prime}$ and by $\psi \circ \pi$ have the same image. Hence $\psi \circ \pi: T^{2} \rightarrow E^{\prime}$ lifts to a diffeomorphism $f: T^{2} \rightarrow T^{2}$ such that $\pi^{\prime} \circ f=\psi \circ \pi$. Since $\tau$ (resp. $\tau^{\prime}$ ) takes $x \in T^{2}$ to the unique other point in $\pi^{-1}(\pi(x))$ (resp. $\pi^{\prime-1}\left(\pi^{\prime}(x)\right)$ ) it follows that $\tau^{\prime} \circ f=f \circ \tau$, so $\tau^{\prime}$ and $\tau$ are indeed conjugate.

In light of this, identifying $T^{2}=\mathbb{R}^{2} / \mathbb{Z}^{2}$ and conjugating $\tau_{1}, \tau_{2}, \phi$ by some diffeomorphism, we can assume that $\tau_{1}([x, y])=[x+1 / 2, y]$ (where $[x, y] \in T^{2}$ is the equivalence class of $(x, y) \in \mathbb{R}^{2}$ under the relations $(x+1, y) \sim(x, y+1) \sim$ $(x, y))$. By the previous paragraph, since $\phi^{-1} \circ \tau_{2} \circ \phi$ and $\tau_{1}$ are free involutions, there is some $\alpha \in \operatorname{Diff}\left(T^{2}\right)$ such that $\phi^{-1} \circ \tau_{2} \circ \phi=\alpha^{-1} \circ \tau_{1} \circ \alpha$. Now $\alpha$ is isotopic to some linear diffeomorphism $A=\left(\begin{array}{ll}a & b \\ c & d\end{array}\right) \in \operatorname{SL}(2 ; \mathbb{Z})$; say $A=\alpha \circ f_{1}$ where $\left\{f_{t}\right\}_{t \in[0,1]}$ is 
a smooth family of diffeomorphisms such that $f_{0}=1$. Then where $\phi^{\prime}=\phi \circ f_{1}, \phi$ is isotopic to $\phi^{\prime}$ and we have $\phi^{\prime-1} \circ \tau_{2} \circ \phi^{\prime}=A^{-1} \circ \tau_{1} \circ A$.

Now since $\tau_{1}([x, y])=[x+1 / 2, y]$, one easily computes

$$
A^{-1} \circ \tau_{1} \circ A \circ \tau_{1}([x, y])=\left[x+\frac{d+1}{2}, y-\frac{c}{2}\right],
$$

which defines the identity if $c$ is even (forcing $d$ to be odd since $A \in \operatorname{SL}(2 ; \mathbb{Z})$ ) and a free involution if $c$ is odd.

Theorem 3.9. Let $\pi_{i}: X_{i} \rightarrow T^{2}(i=1,2)$ be $S^{2}$-bundles over $T^{2}, F_{i} \subset X_{i}$ embedded tori with the property that $\left.\pi_{i}\right|_{F_{i}}$ is an unramified double cover. Let $v_{i}$ be tubular neighborhoods of $F_{i}$ (each identified with $D^{2} \times F_{i}$ ), and let $\Phi: \partial v_{1} \rightarrow \partial v_{2}$ be a diffeomorphism which (viewing $\partial v_{i}$ as an $S^{1}$-bundle over $F_{i}$ ) covers some diffeomorphism $\phi: F_{1} \rightarrow F_{2}$. Then the normal connect sum

$$
\left(X_{1} \backslash v_{1}\right) \cup_{\partial v_{1} \sim} \sim_{\Phi} \partial v_{2}\left(X_{2} \backslash v_{2}\right)
$$

is diffeomorphic to the total space of a $T^{2}$-bundle over $T^{2}$.

Proof. First, note that (after performing isotopies which do not change the diffeomorphism type of the normal connect sum), we can assume that, for $i=1,2$, the $S^{2}$ bundle projection $\pi_{i}$ is constant on each fiber of the disc bundle projection $v_{i} \rightarrow F_{i}$, and that (using Lemma 3.8) $\phi^{-1} \circ \tau_{2} \circ \phi \circ \tau_{1}$ is either the identity or a free involution, where $\tau_{i}: F_{i} \rightarrow F_{i}$ is the deck transformation induced by the cover $\left.\pi_{i}\right|_{F_{i}}$. Let $Z=\left(X_{1} \backslash v_{1}\right) \cup_{\partial v_{1} \sim \sim_{\Phi} \partial v_{2}}\left(X_{2} \backslash v_{2}\right)$.

Suppose that $\phi^{-1} \circ \tau_{2} \circ \phi \circ \tau_{1}$ is the identity. We define a bundle map $\pi: Z \rightarrow T^{2}$ as follows. If $x \in X_{1} \backslash v_{1} \subset Z$, put $\pi(x)=\pi_{1}(x)$. If $x \in X_{2} \backslash v_{2} \subset Z$, then there are two points $x_{2}, \tau_{2}\left(x_{2}\right) \in F_{2} \cap \pi_{2}^{-1}\left(\left\{\pi_{2}(x)\right\}\right)$, and since $\phi^{-1} \circ \tau_{2} \circ \phi \circ \tau_{1}$ is the identity we have $\tau_{1}\left(\phi^{-1}\left(x_{2}\right)\right)=\phi^{-1}\left(\tau_{2}\left(x_{2}\right)\right)$, so that $\pi_{1}\left(\phi^{-1}\left(x_{2}\right)\right)=\pi_{1}\left(\phi^{-1}\left(\tau_{2}\left(x_{2}\right)\right)\right)$ and we set

$$
\pi(x)=\pi_{1}\left(\phi^{-1}\left(x_{2}\right)\right)=\pi_{1}\left(\phi^{-1}\left(\tau_{2}\left(x_{2}\right)\right)\right) .
$$

Since, for each $p \in F_{i}$, the fiber of the circle bundle $\partial v_{i} \rightarrow F_{i}$ over $p$ is mapped by $\pi_{i}$ to $\pi_{i}(p)$, our map $\pi$ is defined consistently on the identified boundary components $\partial v_{1}, \partial v_{2}$ in $Z=\left(X_{1} \backslash v_{1}\right) \cup_{\partial \nu_{1} \sim_{\Phi} \partial v_{2}}\left(X_{2} \backslash v_{2}\right)$. One easily sees that $\pi: Z \rightarrow T^{2}$ is a $T^{2}$-fibration; the point here is that since $\phi^{-1} \circ \tau_{2} \circ \phi \circ \tau_{1}=1, \phi: F_{1} \rightarrow F_{2}$ descends to a map $f: T^{2} \rightarrow T^{2}$ such that $\left.\pi_{2}\right|_{F_{2}} \circ \phi=\left.f \circ \pi_{1}\right|_{F_{1}}$; the fiber of $\pi$ over $t \in T^{2}$ is formed by gluing the annulus $\pi_{1}^{-1}(\{t\}) \cap\left(X_{1} \backslash v_{1}\right)$ to the annulus $\pi_{2}^{-1}(\{f(t)\}) \cap\left(X_{2} \backslash v_{2}\right)$.

It remains to consider the case that $\phi^{-1} \circ \tau_{2} \circ \phi \circ \tau_{1}$ is a free involution. Then $\phi^{-1} \circ \tau_{2} \circ \phi \circ \tau_{1}$ commutes with $\tau_{1}$ and their composition (namely $\phi^{-1} \circ \tau_{2} \circ \phi$ ) is also a free involution. Let $E=F_{1} /\left\langle\tau_{1}, \phi^{-1} \circ \tau_{2} \circ \phi \circ \tau_{1}\right\rangle$ and let $p: F_{1} \rightarrow E$ be the projection 
(which is an unramified quadruple covering of a torus by a torus). We shall define a torus fibration $\pi: Z \rightarrow E$ rather similarly to the previous case, except that here the fibers will be formed by gluing four annuli rather than two. If $x \in X_{1} \backslash v_{1} \subset Z$, set $\pi(x)=p\left(x_{1}\right)$ where $x_{1} \in F_{1} \cap \pi_{1}^{-1}\left(\left\{\pi_{1}(x)\right\}\right)$; this is a coherent definition since the two elements of $\pi_{1}^{-1}\left(\left\{\pi_{1}(x)\right\}\right)$ are intertwined by $\tau_{1}$. If $x \in X_{2} \backslash \nu_{2} \subset Z$, we intend to set $\pi(x)=p\left(\phi^{-1}\left(x_{2}\right)\right)$ where $x_{2} \in F_{2} \cap \pi_{2}^{-1}\left(\left\{\pi_{2}(x)\right\}\right)$; we need to see that the two possible choices of $x_{2}$ (either of which is taken to the other by $\tau_{2}$ ) give the same value for $p(x)$. In other words, we need to check that if $x_{2} \in F_{2}$ then $\left.p\left(\phi^{-1}\left(x_{2}\right)\right)\right)=p\left(\phi^{-1}\left(\tau_{2}\left(x_{2}\right)\right)\right)$. Now since $\phi^{-1} \circ \tau_{2} \circ \phi \circ \tau_{1}$ has order 2 ,

$$
\phi^{-1}\left(\tau_{2}\left(\phi\left(\tau_{1}\left(\phi^{-1}\left(x_{2}\right)\right)\right)\right)\right)=\tau_{1}\left(\phi^{-1}\left(\tau_{2}\left(x_{2}\right)\right)\right)
$$

so since $E$ is the quotient of $F_{1}$ by $\tau_{1}$ and $\phi^{-1} \circ \tau_{2} \circ \phi \circ \tau_{1}$ it is indeed the case that $\left.p\left(\phi^{-1}\left(x_{2}\right)\right)\right)=p\left(\phi^{-1}\left(\tau_{2}\left(x_{2}\right)\right)\right)$ for each $x_{2} \in F_{2}$. We have thus defined $\pi: Z \rightarrow E$; it is again easily seen to be a torus bundle, with its fibers of the shape

$$
\frac{A_{0} \bigsqcup A_{1} \bigsqcup A_{2} \bigsqcup A_{3}}{\partial_{+} A_{i} \sim \partial_{-} A_{i+1} \quad(i \in \mathbb{Z} / 4 \mathbb{Z})}
$$

where $A_{0}$ and $A_{2}$ are annulus fibers of $\left.\pi_{2}\right|_{X_{2} \backslash \nu_{2}}$ and $A_{1}$ and $A_{3}$ are annulus fibers of $\left.\pi_{1}\right|_{X_{1} \backslash \nu_{1}}$ (the fact that $\phi^{-1} \circ \tau_{2} \circ \phi \circ \tau_{1}$ is free serves to ensure that, in each of these torus fibers, $A_{0}$ and $A_{2}$ are distinct, as are $A_{1}$ and $A_{3}$ ).

This shows that any symplectic 4-manifold obtained as the symplectic sum of two $S^{2}$-bundles over $T^{2}$ along a pair of bi-sections is diffeomorphic to a $T^{2}$-bundle over $T^{2}$. In fact, we can be quite specific about which $T^{2}$-bundles over $T^{2}$ are obtained in this fashion. $T^{2}$-bundles over $T^{2}$ were classified in [24]; in particular, Theorem 5 of that paper shows that the total spaces of such bundles are distinguished from one another up to diffeomorphism by their fundamental groups. As such, finding the diffeomorphism type of the manifold $\left(X_{1} \backslash v_{1}\right) \cup_{\partial \nu_{1} \sim_{\Phi} \partial v_{2}}\left(X_{2} \backslash v_{2}\right)$ in Theorem 3.9 is just a matter of applying van Kampen's theorem.

We know that, for $i=1,2$, the manifold $X_{i} \backslash v_{i}$ is diffeomorphic to one of the manifolds $Y_{0}$ or $Y_{1}$ of (3), (4); more specifically, if $X_{i}$ is diffeomorphic to $S^{2} \times T^{2}$ then $X_{i} \backslash v_{i} \cong Y_{0}$, and otherwise $X_{i} \backslash v_{i} \cong Y_{1}$. Note that

$$
\pi_{1}\left(Y_{j}\right)=\frac{\langle\alpha, \beta, m\rangle}{\alpha^{-1} m \alpha=m^{-1}, \beta m=m \beta, \alpha \beta \alpha^{-1} \beta^{-1}=m^{j}} \quad(j=0,1),
$$

with $\partial Y_{j}$ being spanned by the subgroup generated by $\alpha^{2}, \beta, m . m$ here is the generator of the fundamental group of the annulus fiber of the bundle map $Y_{j} \rightarrow T^{2}$. Where $F$ denotes the torus $F_{1}$ or $F_{2}$ whose neighborhood we have removed from $X_{1}$ or $X_{2}$ to get $Y_{j}$, we have a trivial circle bundle $p_{j}: \partial Y_{j} \rightarrow F$ whose action on $\pi_{1}$ has kernel $\langle m\rangle$. There is, of course, some flexibility in the choice of the generators: first, 
we get the same presentation if we replace $\beta$ by $\beta m^{j}$ and then $m$ by $m^{-1}$; secondly, if $\left(\begin{array}{cc}p & q \\ r & s\end{array}\right) \in \mathrm{SL}(2, \mathbb{Z})$ and $q$ is even (so that $p$ and $s$ are odd; say $s=2 t+1$ ), then we get the same presentation by replacing $\alpha$ with $\alpha^{\prime}=\alpha^{p} \beta^{r}$ and $\beta$ with $\beta^{\prime}=\alpha^{q} \beta^{s} m^{j t}$.

One convenient consequence of this is that if $\gamma \in \pi_{1}\left(\partial Y_{j}\right)$ is any element with the property that $\left(p_{j}\right)_{*} \gamma$ is primitive in $\mathbb{Z}^{2}=\pi_{1}(F)$ then the generators $\alpha, \beta$ in the presentation (5) may be chosen so that $\gamma$ takes one of the forms

$$
\gamma=\alpha^{2} \beta^{2 c} m^{e} \quad \text { or } \quad \gamma=\alpha^{2 a} \beta m^{e} .
$$

We now consider the manifold resulting from gluing two of these manifolds $Y_{j}, Y_{k}$ $(j, k \in\{0,1\})$ together along their boundaries in a way consistent with the symplectic sum operation. Now in terms of bases $\left\{\alpha_{1}^{2}, \beta_{1}, m_{1}\right\},\left\{\alpha_{2}^{2}, \beta_{2}, m_{2}\right\}$ for the fundamental groups of the boundaries $\partial Y_{j}$ and $\partial Y_{k}$ respectively, since the gluing map $\Phi$ is required to cover an isomorphism of the normal bundles it will identify $m_{1}$ with $m_{2}$ (possibly after replacing one of the $m_{i}$ with its inverse, which as mentioned earlier can be done without affecting the presentation (5) at the cost of possibly multiplying $\beta_{i}$ by $m_{i}$ ); also $\alpha_{1}^{2}$, since it projects via $p_{j}$ to a primitive element in $\pi_{1}(F)$, will be taken to some element in the fundamental group of $\partial Y_{k}$ which likewise projects via $p_{k}$ to a primitive element. Hence by the remark at the end of the previous paragraph, possibly after renaming the generators $\alpha_{2}, \beta_{2}$, and $m_{2}$ in the presentation of $\pi_{1}\left(Y_{k}\right)$, the action of the gluing map on the fundamental groups of the boundaries in terms of the bases $\left\{\alpha_{i}^{2}, \beta_{i}, m_{i}\right\}$ takes one of the forms

$$
\left(\begin{array}{ccc}
1 & b & 0 \\
2 c & d & 0 \\
e & f & 1
\end{array}\right) \quad(d-2 b c=1) \quad \text { or } \quad\left(\begin{array}{ccc}
a & b & 0 \\
1 & d & 0 \\
e & f & 1
\end{array}\right) \quad(a d-b=1) .
$$

Hence van Kampen's theorem gives the fundamental group of the glued manifold $Y_{j} \cup_{\Phi} Y_{k}$ as either

$$
\begin{gathered}
\pi_{1}\left(Y_{j} \cup_{\Phi} Y_{k}\right)=\frac{\left\langle\alpha_{1}, \beta_{1}, \alpha_{2}, \beta_{2}, m\right\rangle}{\alpha_{1}^{-1} m \alpha_{1}=\alpha_{2}^{-1} m \alpha_{2}=m^{-1}, \beta_{1} m=m \beta_{1}, \beta_{2} m=m \beta_{2},} \\
\alpha_{1} \beta_{1} \alpha_{1}^{-1} \beta_{1}^{-1}=m^{j}, \alpha_{2} \beta_{2} \alpha_{2}^{-1} \beta_{2}^{-1}=m^{k}, \\
\alpha_{1}^{2}=\alpha_{2}^{2} \beta_{2}^{2 c} m^{e}, \beta_{1}=\alpha_{2}^{2 b} \beta_{2}^{d} m^{f}
\end{gathered}
$$

or

$$
\begin{gathered}
\pi_{1}\left(Y_{j} \cup_{\Phi} Y_{k}\right)=\frac{\left\langle\alpha_{1}, \beta_{1}, \alpha_{2}, \beta_{2}, m\right\rangle}{\alpha_{1}^{-1} m \alpha_{1}=\alpha_{2}^{-1} m \alpha_{2}=m^{-1}, \beta_{1} m=m \beta_{1}, \beta_{2} m=m \beta_{2},} \\
\alpha_{1} \beta_{1} \alpha_{1}^{-1} \beta_{1}^{-1}=m^{j}, \alpha_{2} \beta_{2} \alpha_{2}^{-1} \beta_{2}^{-1}=m^{k}, \\
\alpha_{1}^{2}=\alpha_{2}^{2 a} \beta_{2} m^{e}, \beta_{1}=\alpha_{2}^{2 b} \beta_{2}^{d} m^{f}
\end{gathered}
$$

The reader may verify that the group on the right hand side of (6) may be rewritten, 
by identifying $\gamma=\alpha_{1}^{-1} \alpha_{2} \beta_{2}^{c}$, as

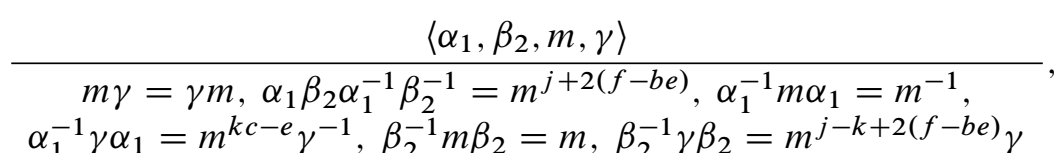

which we recognize as the fundamental group of the $T^{2}$-bundle over $T^{2}$ given in the notation of the introduction as

$$
M\left(\left(\begin{array}{cc}
-1 & k c-e \\
0 & -1
\end{array}\right),\left(\begin{array}{cc}
1 & j-k+2(f-b e) \\
0 & 1
\end{array}\right) ;\left(\begin{array}{c}
j+2(f-b e) \\
0
\end{array}\right)\right)
$$

Similarly, the group on the right hand side of (7) may be identified, by taking $\sigma=$ $\alpha_{1} \alpha_{2} \alpha_{1}^{-1} \alpha_{2}^{-1}$ and then using the relations $\alpha_{1} \alpha_{2}^{2}=\alpha_{2}^{2} \alpha_{1} m^{j+2(f-d e)}$ and $\alpha_{2} \alpha_{1}^{2}=$ $\alpha_{1}^{2} \alpha_{2} m^{2 e-k}$ to obtain commutation relations between the $\alpha_{i}$ and $\sigma$, as

$$
\begin{gathered}
\left\langle\alpha_{1}, \alpha_{2}, m, \sigma\right\rangle \\
m \sigma=\sigma m, \alpha_{1} \alpha_{2} \alpha_{1}^{-1} \alpha_{2}^{-1}=\sigma, \alpha_{1}^{-1} m \alpha_{1}=m^{-1} \\
\alpha_{1}^{-1} \sigma \alpha_{1}=m^{k-2 e} \sigma^{-1}, \alpha_{2}^{-1} m \alpha_{2}=m^{-1}, \alpha_{2}^{-1} \sigma \alpha_{2}=m^{j+2(f-d e)} \sigma^{-1}
\end{gathered}
$$

which is precisely the fundamental group of the $T^{2}$-bundle over $T^{2}$

$$
M\left(\left(\begin{array}{cc}
-1 & k-2 e \\
0 & -1
\end{array}\right),\left(\begin{array}{cc}
-1 & j+2(f-d e) \\
0 & -1
\end{array}\right) ;\left(\begin{array}{l}
0 \\
1
\end{array}\right)\right) .
$$

Now by changing the basis for the homology of the base by $(\underset{r}{p} \underset{s}{q}) \in \operatorname{SL}(2 ; \mathbb{Z})$, a $T^{2}$-bundle over $T^{2}$ of form $M(A, B ; \vec{v})$ may be equated with $M\left(A^{p} B^{r}, A^{q} B^{s} ; \vec{v}\right)$; also, the bundles $M(A, B ; \vec{v})$ and $M\left(A, B ; \vec{v}^{\prime}\right)$ are equivalent if $\vec{v}^{\prime}-\vec{v}$ lies in the submodule of $\mathbb{Z}^{2}$ spanned by the columns of $A-I$ and $B-I$ (where $I$ is the identity; these statements are proven in Proposition 2 of [24]). As such, given a bundle of form

$$
M\left(\left(\begin{array}{cc}
-1 & \delta \\
0 & -1
\end{array}\right),\left(\begin{array}{ll}
1 & \zeta \\
0 & 1
\end{array}\right) ;\left(\begin{array}{c}
j+2 x \\
0
\end{array}\right)\right)
$$

by letting $z=\operatorname{gcd}(\delta, \zeta), p=\zeta / z, r=\delta / z$, and (since $p$ and $r$ are then relatively prime) $q$ and $s$ be such that $p s-q r=1$, so that $-q \delta+s \zeta=z$, we obtain

$$
M\left(\left(\begin{array}{cc}
-1 & \delta \\
0 & -1
\end{array}\right),\left(\begin{array}{cc}
1 & \zeta \\
0 & 1
\end{array}\right) ;\left(\begin{array}{c}
j+2 x \\
0
\end{array}\right)\right)=M\left((-1)^{p} I,(-1)^{q}\left(\begin{array}{ll}
1 & z \\
0 & 1
\end{array}\right) ;\left(\begin{array}{l}
j \\
0
\end{array}\right)\right)
$$

(note also that $p$ and $q$ cannot both be even since $p s-q r=1$, and if $p$ is odd then a further basis change for the homology of the base identifies $M(-I,-A ; \vec{v})$ with $M(-I, A ; \vec{v}))$. This gives rise to the following list of possibilities for the diffeomorphism type of $Y_{j} \cup_{\Phi} Y_{k}$ when its fundamental group is given by (6): 


\begin{tabular}{l|l|l}
$j$ & $k$ & possible diffeomorphism types of $Y_{j} \cup_{\Phi} Y_{k}$ \\
\hline 0 & 0 & $M\left(I,\left(\begin{array}{cc}-1 & z \\
0 & -1\end{array}\right) ;\left(\begin{array}{l}0 \\
0\end{array}\right)\right), M\left(-I,\left(\begin{array}{cc}1 & 2 y \\
0 & 1\end{array}\right) ;\left(\begin{array}{l}0 \\
0\end{array}\right)\right) \quad(y, z \in \mathbb{Z})$ \\
\hline 0 & 1 & $M\left(-I,\left(\begin{array}{cc}1 & 2 y+1 \\
0 & 1\end{array}\right) ;\left(\begin{array}{l}0 \\
0\end{array}\right)\right) \quad(y \in \mathbb{Z})$ \\
\hline 1 & 1 & $M\left(I,\left(\begin{array}{cc}-1 & z \\
0 & -1\end{array}\right) ;\left(\begin{array}{l}1 \\
0\end{array}\right)\right), M\left(-I,\left(\begin{array}{cc}1 & 2 y \\
0 & 1\end{array}\right) ;\left(\begin{array}{l}1 \\
0\end{array}\right)\right) \quad(y, z \in \mathbb{Z})$
\end{tabular}

Similarly, a bundle of form

$$
M\left(\left(\begin{array}{cc}
-1 & \delta \\
0 & -1
\end{array}\right),\left(\begin{array}{cc}
-1 & \zeta \\
0 & -1
\end{array}\right) ;\left(\begin{array}{l}
0 \\
1
\end{array}\right)\right)
$$

is equivalent to

$$
M\left((-1)^{p+r} I,(-1)^{q+s}\left(\begin{array}{ll}
1 & z \\
0 & 1
\end{array}\right) ;\left(\begin{array}{l}
0 \\
1
\end{array}\right)\right)
$$

(where $z=\operatorname{gcd}(\delta, \zeta), p=\zeta / z, r=-\delta / z$, and $p s-q r=1$ ). From this, we deduce the following list of possibilities for the diffeomorphism type of $Y_{j} \cup_{\Phi} Y_{k}$ when its fundamental group is given by (7):

\begin{tabular}{l|l|l}
$j$ & $k$ & possible diffeomorphism types of $Y_{j} \cup_{\Phi} Y_{k}$ \\
\hline 0 & 0 & $M\left(I,\left(\begin{array}{cc}-1 & 2 y \\
0 & -1\end{array}\right) ;\left(\begin{array}{l}0 \\
1\end{array}\right)\right), M\left(-I,\left(\begin{array}{cc}1 & 2 y \\
0 & 1\end{array}\right) ;\left(\begin{array}{l}0 \\
1\end{array}\right)\right) \quad(y \in \mathbb{Z})$ \\
\hline 0 & 1 & $M\left(-I,\left(\begin{array}{cc}1 & 2 y+1 \\
0 & 1\end{array}\right) ;\left(\begin{array}{l}0 \\
1\end{array}\right)\right) \quad(y \in \mathbb{Z})$ \\
\hline 1 & 1 & $M\left(I,\left(\begin{array}{cc}-1 & 2 y+1 \\
0 & -1\end{array}\right) ;\left(\begin{array}{l}0 \\
1\end{array}\right)\right) \quad(y \in \mathbb{Z})$
\end{tabular}

In both of the above tables, it is easy to see that any of the indicated diffeomorphism types can in fact be realized by means of an appropriate choice of the gluing map $\Phi$. Since if $X$ is a ruled surface over $T^{2}$ and $F \subset X$ is an embedded representative of $-\mathrm{PD}\left(\kappa_{X}\right)$, we have seen that $X \backslash \nu F \cong Y_{0}$ if $X=S^{2} \times T^{2}$ and $X \backslash \nu F \cong Y_{1}$ if $X=S^{2} \tilde{X} T^{2}$, this completes the proof that the diffeomorphism types of the symplectic sums in question are as claimed in the introduction. 


\section{References}

[1] S. Bauer, Almost complex 4-manifolds with vanishing first Chern class. J. Differential Geom. 79 (2008), 25-32. Zbl 1144.57027 MR 2414748

[2] R. Fintushel and R. Stern, Rational blowdowns of smooth four-manifolds. J. Differential Geom. 46 (1997), 181-235. Zbl 0896.57022 MR 1484044

[3] R. Fintushel and R. Stern, Knots, links and 4-manifolds. Invent. Math. 134 (1998), 363-400. Zbl 0914.57015 MR 1650308

[4] R. Fintushel and R. Stern, Symplectic surfaces in a fixed homology class. J. Differential Geom. 52 (1999), 203-222. Zbl 0981.53085 MR 1758295

[5] H. Geiges, Symplectic structures on $T^{2}$-bundles over $T^{2}$. Duke Math. J. 67 (1992), 539-555. Zbl 0763.53037 MR 1181312

[6] R. Gompf, Nuclei of elliptic surfaces. Topology 30 (1991), 479-511. Zbl 0732.57010 MR 1113691

[7] R. Gompf, A new construction of symplectic manifolds. Ann. of Math. 142 (1995), 527-595. Zbl 0849.53027 MR 1356781

[8] R. Gompf and A. Stipsicz, 4-manifolds and Kirby calculus. Grad. Stud. Math. 20, Amer. Math. Soc., Providence, RI, 1999. Zbl 0933.57020 MR 1707327

[9] P. Griffiths and J. Harris, Principles of Algebraic Geometry. Wiley, New York 1978. Zbl 0408.14001 MR 0507725

[10] M. Gromov, Pseudo-holomorphic curves in symplectic manifolds. Invent. Math. 82 (1985), 307-347. Zbl 0592.53025 MR 0809718

[11] L. Guillou and A. Marin, Commentaires sur les quatres articles précédentes de V. A. Rohlin. In À la recherche de la topologie perdue, L. Guillou and A. Marin, eds., Birkhäuser, Boston 1986, 25-97.

[12] E. Ionel and T. Parker, The symplectic sum formula for Gromov-Witten invariants. Ann. of Math. 159 (2004), 935-1025. Zbl 1075.53092 MR 2113018

[13] F. Lalonde and D. McDuff, The classification of ruled symplectic 4-manifolds. Math. Res. Lett. 3 (1996), 769-778. Zbl 0874.57019 MR 1426534

[14] T. J. Li, Symplectic 4-manifolds of Kodaira dimension zero. J. Differential Geom. 74 (2006), 321-352. Zbl 1105.53068 MR 2259057

[15] T. J. Li, Quaternionic bundles and Betti numbers of symplectic 4-manifolds with Kodaira dimension zero. Internat. Math. Res. Notices 2006 (2006), Article ID 37385, 1-28. Zbl 1129.57037 MR 2264722

[16] T. J. Li and M. Usher, Symplectic forms and surfaces of negative square. J. Symplectic Geom. 4 (2006), 71-91. Zbl 1120.53052 MR 2240213

[17] A. K. Liu, Some new applications of the general wall crossing formula, Gompf's conjecture and its applications. Math. Res. Lett. 3 (1996), 569-585. Zbl 0872.57025 MR 1418572

[18] J. McCarthy and J. Wolfson, Symplectic normal connect sum. Topology 33 (1994), 729-764. Zbl 0812.53033 MR 1293308

[19] D. McDuff, The structure of rational and ruled symplectic 4-manifolds. J. Amer. Math. Soc. 3 (1990), 679-712. Zbl 0723.53019 MR 1168961 
[20] D. McDuff and D. Salamon, A survey of symplectic four-manifolds with $b^{+}=1$. Turkish J. Math. 20 (1996), 47-60. Zbl 0870.57023 MR 1392662

[21] D. McDuff and D. Salamon, Introduction to symplectic topology. Oxford Math. Monogr., Oxford University Press, Oxford 1998. Zbl 1066.53137 MR 1698616

[22] D. McDuff and M. Symington, Associativity properties of the symplectic sum. Math. Res. Lett. 3 (1996), 591-608. Zbl 0877.53025 MR 1418574

[23] H. Seifert, Topologie dreidimensionaler gefaserter Räume. Acta Math. 60 (1933), 147-238; English transl. in Seifert and Threlfall: A textbook of topology, Academic Press, New York 1980. JFM 59.1241.02 Zbl 0006.08304 MR 1555366

[24] K. Sakamoto and S. Fukuhara, Classification of $T^{2}$-bundles over $T^{2}$. Tokyo J. Math. 6 (1983), 311-327. Zbl 0543.55011 MR 0732086

[25] J.-C. Sikorav, The gluing construction for normally generic $J$-holomorphic curves. In Symplectic and contact topology: interactions and perspectives (Toronto, ON/Montreal, QC, 2001), Fields Inst. Commun. 35, Amer. Math. Soc., Providence, RI, 2003, 175-199. Zbl 1109.32301 MR 1969276

[26] C. Taubes, Seiberg-Witten and Gromov invariants for symplectic 4-manifolds. First Int. Press Lect. Ser. 2, International Press, Somerville, MA, 2000. Zbl 0967.57001 MR 1798809

[27] M. Usher, Minimality and symplectic sums. Internat. Math. Res. Notices 2006 (2006), Article ID 49857, 1-17. Zbl 1110.57017 MR 2250015

[28] T. Yagasaki, Homotopy types of diffeomorphism groups of noncompact 2-manifolds. Preprint 2001; arXiv:math.GT/0109183.

Received February 2, 2007

Michael Usher, Department of Mathematics, University of Georgia, Athens, GA 30602, U.S.A.

E-mail: usher@math.uga.edu 